University of South Florida

DIGITAL COMMONS

Digital Commons @ University of

@ UNIVERSITY OF SOUTH FLORIDA

South Florida

School of Geosciences Faculty and Staff

Publications

School of Geosciences

$5-2009$

\title{
Fore-arc Motion and Cocos Ridge Collision in Central America
}

\author{
Peter LaFemina \\ University of Miami \\ Timothy Dixon \\ University of Miami, thd@usf.edu \\ Rob Govers \\ Utrecht University \\ Edmundo Norabuena \\ Instituto Geofisico del Peru \\ Henry Turner \\ University of Arkansas
}

See next page for additional authors

Follow this and additional works at: https://digitalcommons.usf.edu/geo_facpub

Part of the Earth Sciences Commons

\section{Scholar Commons Citation}

LaFemina, Peter; Dixon, Timothy; Govers, Rob; Norabuena, Edmundo; Turner, Henry; Saballos, Armando; Mattioli, Glen; Protti, Marino; and Strauch, Wilfried, "Fore-arc Motion and Cocos Ridge Collision in Central America" (2009). School of Geosciences Faculty and Staff Publications. 440.

https://digitalcommons.usf.edu/geo_facpub/440

This Article is brought to you for free and open access by the School of Geosciences at Digital Commons @ University of South Florida. It has been accepted for inclusion in School of Geosciences Faculty and Staff Publications by an authorized administrator of Digital Commons @ University of South Florida. For more information, please contact digitalcommons@usf.edu. 


\section{Authors}

Peter LaFemina, Timothy Dixon, Rob Govers, Edmundo Norabuena, Henry Turner, Armando Saballos, Glen Mattioli, Marino Protti, and Wilfried Strauch 


\title{
Fore-arc motion and Cocos Ridge collision in Central America
}

\author{
Peter LaFemina \\ Rosenstiel School of Marine and Atmospheric Science, University of Miami, Miami, Florida 33149, USA \\ Now at Department of Geosciences, Pennsylvania State University, University Park, Pennsylvania 16802, USA \\ (plafemina@geosc.psu.edu)
}

Timothy H. Dixon

Rosenstiel School of Marine and Atmospheric Science, University of Miami, Miami, Florida 33149, USA

\section{Rob Govers}

Department of Earth Sciences, Utrecht University, NL-3508 TA Utrecht, Netherlands

\section{Edmundo Norabuena}

Geodesia y Sismotectonica, Instituto Geofisico del Peru, Calle Badajoz 169, Lima, 3, Peru

\section{Henry Turner}

Department of Geosciences, University of Arkansas, Fayetteville, Arkansas 72701, USA

\section{Armando Saballos}

Instituto Nicarguenses de Estudios Territoriales, Frente a Hospital Metrópoli Xolotlán, Apartado Postal 2110, Managua, Nicaragua

\section{Glen Mattioli}

Department of Geosciences, University of Arkansas, Fayetteville, Arkansas 72701, USA

\section{Marino Protti}

OVSICORI, UNA, Apartado 1718-3000, Heredia 3000, Costa Rica

\section{Wilfried Strauch \\ Instituto Nicarguenses de Estudios Territoriales, Frente a Hospital Metrópoli Xolotlán, Apartado Postal 2110, Managua, Nicaragua}

[1] We present the first regional surface velocity field for Central America, showing crustal response to interaction of the Cocos and Caribbean plates. Elastic half-space models for interseismic strain accumulation on the dipping subduction plate boundary fit the GPS data well and show strain accumulation offshore and beneath the Nicoya and Osa peninsulas in Costa Rica but not in Nicaragua. Since large subduction zone earthquakes occur in Nicaragua, we suggest that interseismic locking in Nicaragua and some other parts of Central America occurs but is mainly shallow, $<20 \mathrm{~km}$ depth, too far offshore to be detected by our on-land GPS measurements. Our data also show significant trench-parallel motion for most of the region, generally interpreted as due to oblique convergence and strong mechanical coupling between subducting and overriding plates. However, trench-parallel motion is also observed in central Costa Rica, where plate convergence is normal to the trench, and in the Nicaraguan fore arc, where 
trench-parallel motion is fast, up to $9 \mathrm{~mm} \mathrm{a}^{-1}$, but mechanical coupling is low. A finite element model of collision (as opposed to subduction) involving the aseismic Cocos Ridge also fits the GPS surface velocity field, most significantly reproducing the pattern of trench-parallel motion. We infer that buoyant, thickened CNS-2-Cocos Ridge crust resists normal subduction and instead acts as an indenter to the Caribbean plate, driving crustal shortening in southern Costa Rica and contributing to trench-parallel fore-arc motion in Costa Rica and perhaps Nicaragua as a type of tectonic escape.

Components: 13,289 words, 11 figures, 3 tables.

Keywords: Central America; earthquake cycle; GPS; fore arc.

Index Terms: 8104 Tectonophysics: Continental margins: convergent; 1242 Geodesy and Gravity: Seismic cycle related deformations (6924, 7209, 7223, 7230); 8150 Tectonophysics: Plate boundary: general (3040).

Received 21 July 2008; Revised 9 March 2009; Accepted 17 March 2009; Published 7 May 2009.

LaFemina, P., T. H. Dixon, R. Govers, E. Norabuena, H. Turner, A. Saballos, G. Mattioli, M. Protti, and W. Strauch (2009), Fore-arc motion and Cocos Ridge collision in Central America, Geochem. Geophys. Geosyst., 10, Q05S14, doi:10.1029/ 2008GC002181.

Theme: Central American Subduction System

Guest Editors: G. Alvarado, K. Hoernle, and E. Silver

\section{Introduction}

[2] In the last 2 decades, space geodetic techniques such as GPS have provided increasingly detailed kinematic descriptions of the deforming upper plate in subduction zones. These descriptions allow inferences about the seismic source and associated processes and longer-term deformation. While it has long been recognized that the surface velocity or strain field at a subduction zone is dominated by elastic, seismic cycle processes, it may also be true that some fraction of geodetically measured displacement involves permanent upper plate deformation, for example translation or rotation of rigid fore-arc blocks [McCaffrey, 2002; Allmendinger et $a l ., 2005]$ or generation of Andean-style mountain belts via crustal shortening in the plate convergence direction.

[3] In this paper we present new GPS data defining the first regional surface velocity field for Central America. We integrate these data with published geophysical and geologic data to investigate elastic and permanent deformation in the region, in particular describing the magnitude and pattern of coupling on the Central American subduction zone and trench-parallel translation of the fore arc. Additionally, we explore the possible driving forces for fore-arc translation. In contrast to previous studies emphasizing the role of slip partitioning caused by oblique convergence and coupling along the plate interface in driving this motion, we suggest an important role for Cocos Ridge collision, whereby the ridge acts as a rigid indenter and arc-parallel fore-arc motion represents tectonic escape.

\section{Tectonics of Central America}

[4] The isthmus of Central America is bounded by five tectonic plates; the North American, Caribbean, South American, Nazca and Cocos plates. Here we investigate the interaction of the Cocos and Caribbean plates and the kinematics of the Central American fore arc. Interaction of the Cocos and Caribbean plates, including interseismic coupling and long-term deformation of the upper plate, may depend on a variety of subduction parameters, including convergence rate, azimuth and obliquity, and slab dip, age and morphology, all of which vary significantly along strike of the Central America subduction zone. The Cocos plate subducts northeastward along the Middle America Trench at rates of $76-91 \mathrm{~mm} \mathrm{a}^{-1}$ relative to the Caribbean plate (Figure 1) [DeMets, 2001]. Relative motion between the Cocos and Nazca plates takes place on the approximately north trending right-lateral Panama, Balboa and Coiba fracture zones (Figure 1).

[5] Convergence azimuth relative to the trench varies along strike; southeast of Nicoya Peninsula, Costa Rica convergence is orthogonal, while along 


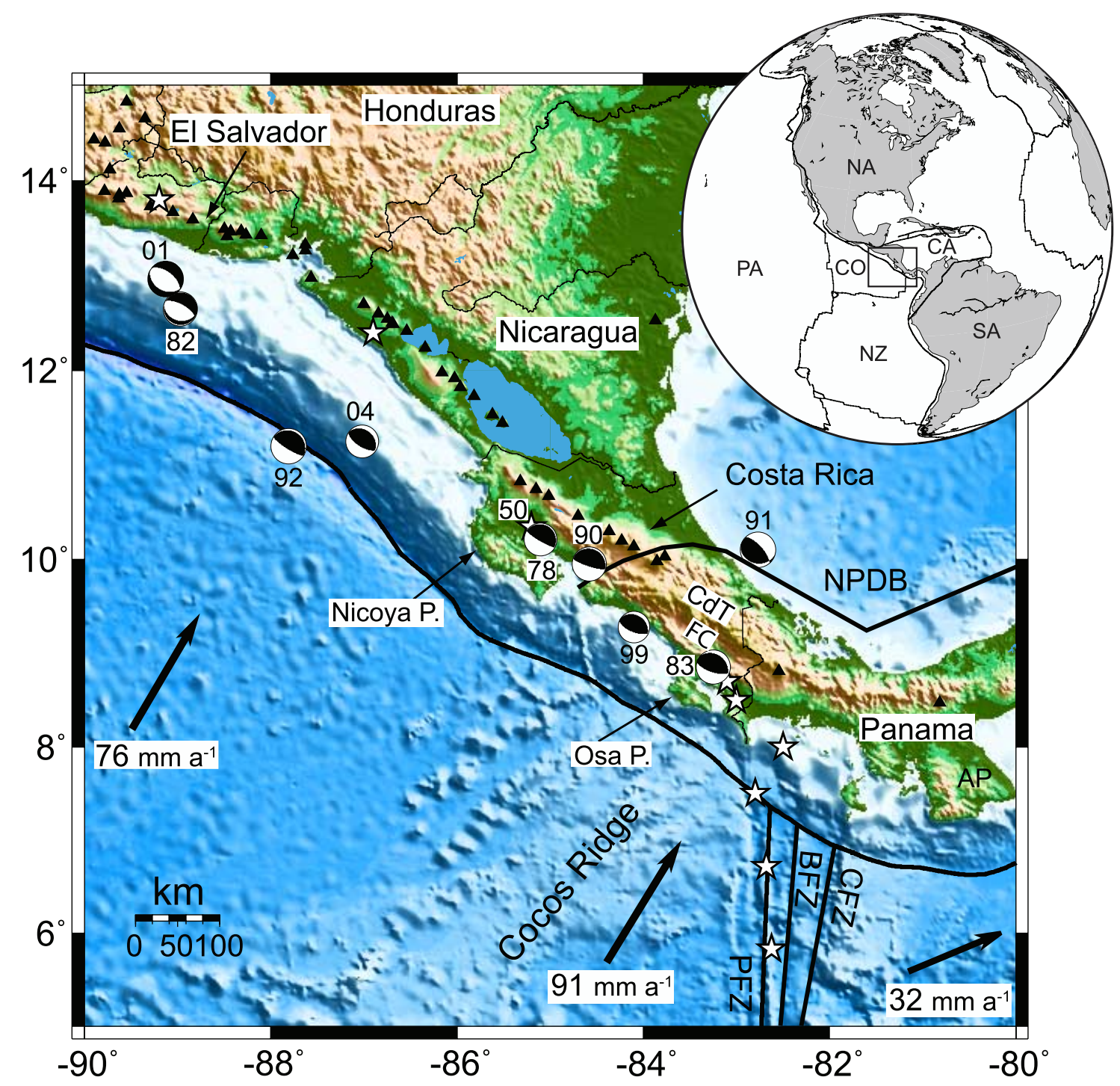

Figure 1. Topographic and bathymetric (GEBCO database) map of Central America. Focal mechanisms and open stars for $M \geq 6$ earthquakes (Global CMT Project and USGS-NEIC; dates for events described in text are noted) indicate active block and plate boundaries [Bird, 2003] (heavy black lines). Black vectors indicate relative rate and azimuth between the Cocos and Caribbean plates [DeMets, 2001] and Nazca-Caribbean plates [Sella et al., 2002]. The Panama (PFZ), Balboa (BFZ), and Coiba (CFZ) Fracture Zones are shown. Black triangles are Holocene volcanoes from the Smithsonian Global Volcanism Program. Thin black lines indicate national boundaries. The Fila Costena (FC) and Cordillera de Talamanca (CdT) are shown in southern Costa Rica, as well as the Azuero Peninsula, Panama (AP). Inset shows tectonic setting of the Central American isthmus, including the plates that interact in this region: North America (NA), Caribbean (CA), South America (SA), Nazca (NZ), Cocos (CO), and Pacific (PA).

the Nicaraguan margin it is up to $25^{\circ}$ oblique to the trench (Figure 2). This convergence obliquity is thought to lead to slip partitioning along the Central American margin and trench-parallel motion of the fore arc at rates as high as $14-15 \mathrm{~mm} \mathrm{a}^{-1}$ [DeMets, 2001; Turner et al., 2007]. This motion was first postulated on the basis of focal mechanisms of intraarc earthquakes [Molnar and Sykes, 1969] and later by the study of the deviation of interplate earthquake slip vectors from the plate convergence direction
[McCaffrey, 1992; 1996; DeMets, 2001] and GPS displacement data [Lundgren et al., 1999; McCaffrey, 2002; Norabuena et al., 2004; Turner et al., 2007]. Unlike the classic slip partitioning case of Sumatra, there is no well-defined margin-parallel strike slip fault. Strain in the fore arc of Costa Rica is accommodated on northwest and northeast trending strike slip and oblique slip conjugate faults [Marshall et al., 2000]. In Nicaragua, fore-arc motion parallel to the trench is accommodated by 


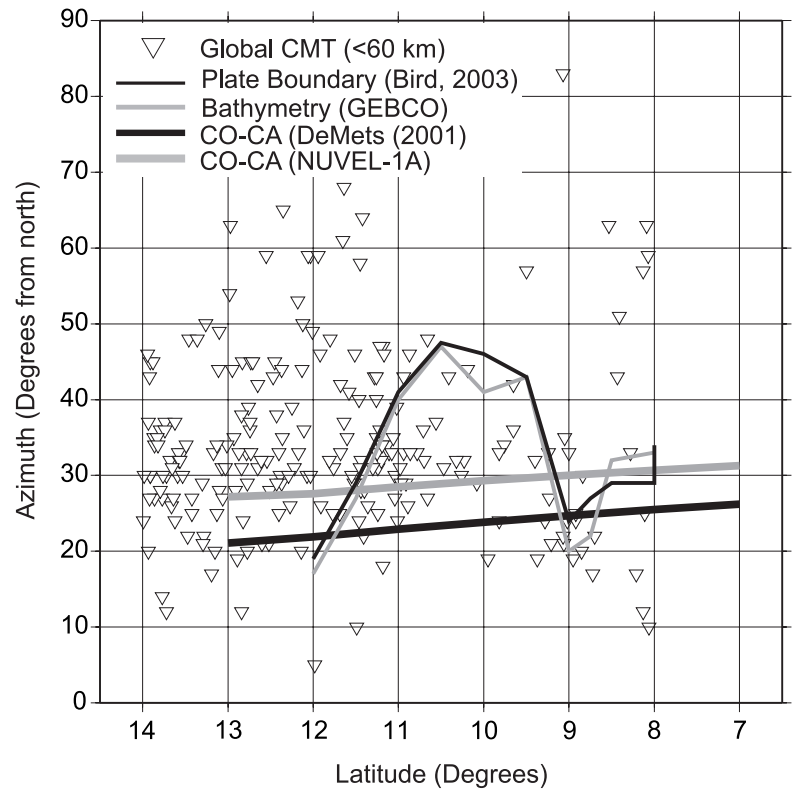

Figure 2. Azimuth versus latitude from $7^{\circ}$ to $14^{\circ} \mathrm{N}$. Thick lines indicate the azimuth of Cocos-Caribbean relative convergence using NUVEL-1A (gray) and DeMets [2001] (black). Earthquake slip vectors (inverted triangles) indicate partitioning of relative plate motion between the Middle America plate boundary and the fore arc. The normal to the Middle America Trench was estimated using the GEBCO bathymetric data set (thin gray line) and plate boundary model of Bird [2003] (thin black line) and shows orthogonal convergence south of Nicoya Peninsula $\left(\sim 9^{\circ} \mathrm{N}\right)$ and up to $25^{\circ}$ obliquity in Nicaragua $\left(\sim 10.5^{\circ} \mathrm{N}\right)$.

northeast striking (arc-normal) left-lateral faults, block rotation, and east-west extension [McCaffrey, 1992, 1996; La Femina et al., 2002]. In El Salvador, fore-arc motion appears to be accommodated along northwest trending (arc parallel) right-lateral strike slip faults [Corti et al., 2005], although more complex fault geometries (e.g., margin-normal faults as in Nicaragua and Costa Rica) cannot be ruled out [Carr, 1976].

[6] The Cocos plate offshore Central America has a complex history that correlates with slab dip [Protti et al., 1995] and may correlate with distribution of plate coupling and upper plate deformation. Northwest of the Nicoya Peninsula, Cocos plate lithosphere formed at the East Pacific Rise (EPR) $\geq 23 \mathrm{Ma}$ (Figure 3a). Cocos lithosphere offshore Nicoya Peninsula and to the southeast formed at the Cocos-Nazca spreading (CNS) center and records two ridge jumps, CNS-1 (23 Ma) and CNS-2 (19 Ma) [Barckhausen et al., 2001]. CNS-2 lithosphere decreases in age southward from $19 \mathrm{Ma}$ to $15 \mathrm{Ma}$, and includes the overprinted $13-14.5 \mathrm{Ma}$ aseismic Cocos Ridge and seamount domain [Werner et al., 1999]. This along-strike variation in Cocos plate age results in variable Cocos slab thermal structure [Spinelli and Saffer, 2004], which has been linked to seismogenesis along the Nicoya segment [Newman et al., 2002; Norabuena et al., 2004; DeShon et al., 2006].

[7] The Cocos Ridge stands $\geq 2 \mathrm{~km}$ above surrounding seafloor and sits atop CNS-2 crust that can exceed $20 \mathrm{~km}$ thickness, roughly double the thickness of standard oceanic crust (Figures 3a and 3b) [Walther, 2003]. The Cocos Ridge, subparallel seamount chains and triple junction traces are oriented $\leq 10^{\circ}$ clockwise from the Cocos-Caribbean relative plate motion vector $\left(\sim \mathrm{N} 24^{\circ} \mathrm{E}\right)$, which results in northwest migration of these features relative to the upper plate [Barckhausen et al., 2001]. CNS-2 and Cocos Ridge crust abuts the seismically active Panama, Balboa and Coiba fracture zones and is juxtaposed against the Nazca plate composed of CNS-1 crust overprinted with the $\geq 15$ Ma Coiba Ridge (Figure 3a) [Werner et al., 1999]. The north trending Cocos-Nazca plate boundary migrates southeastward relative to Caribbean plate at $\sim 35 \mathrm{~mm} \mathrm{a}^{-1}$.

[8] The along-strike variability in age and bathymetry correlates with the dip of the subducting Cocos plate offshore Central America. Local and global seismic tomography, seismic reflection and earthquake studies indicate that:

[9] 1. The dip and depth of the Wadati-Benioff zone decreases from Nicaragua to Costa Rica, with significant changes across the EPR and CNS boundaries. The slab is contorted along the EPR to CNS-1 boundary [Protti et al., 1994]. DeShon et al. [2006] suggest $\sim 5 \mathrm{~km}$ vertical offset in relocated plate interface microseismicity across the EPR to CNS-1 boundary;

[10] 2. There is no seismically defined slab northeast (inboard) of Cocos Ridge or Nazca plate at depths $>60 \mathrm{~km}$ [Vergara Munoz, 1988; Protti et al., 1994];

[11] 3. Historical, large magnitude plate interface earthquakes $\left(M_{w}>7\right)$ may correlate with the locations of subducted seamounts or bathymetric features (e.g., 1992 Nicaragua [McIntosh et al., 2007], 1950 and 1990 Nicoya [Husen et al., 2002], 1983 Osa [Adamek et al., 1987], and 1999 Quepos [Bilek et al., 2003] events) (Figure 1). Seamount subduction has also caused extensive subduction erosion of the outer fore arc offshore Costa Rica [Ranero and von Huene, 2000] and uplift of the 

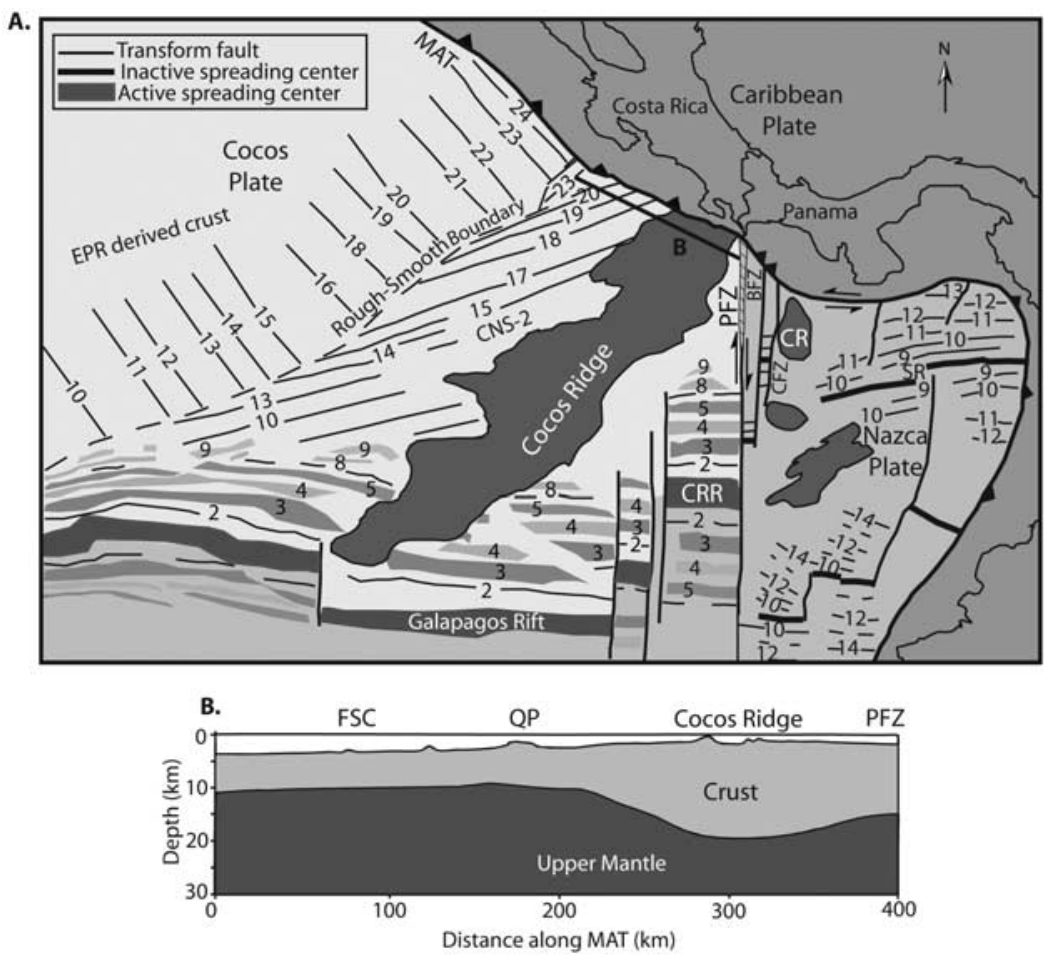

Figure 3. (a) Tectonic map identifying magnetic anomalies on Cocos and Nazca plates from Lonsdale and Klitgord [1978], Lowrie et al. [1979], Hardy [1991], Meschede et al. [1998], Barckhausen et al. [2001], MacMillan et al. [2004], and Lonsdale [2005]. East Pacific Rise (EPR); Cocos Nazca spreading center (CNS); Coiba Ridge (CR); Costa Rica Rift (CRR); Middle America Trench (MAT); Panama (PFZ), Balboa (BFZ), and Coiba (CFZ) fracture zones; and Sandra Rift (SR) are shown. Black line labeled B indicates cross section shown in Figure 3b. (b) Upper mantle and crustal structure of the Cocos plate from von Huene et al. [2000] and Walther [2003]. Note thickened $(>20 \mathrm{~km})$ crust beneath Cocos Ridge. The bathymetric features Fisher seamount chain (FSC) and Quepos plateau (QP) are also shown.

Nicoya and Osa peninsulas and Quepos region [Gardner et al., 1992; Fisher et al., 1998; Gardner et al., 2001; Sak et al., 2004].

[12] 4. The Caribbean plate is underthrusting Central America along the North Panama Deformed Belt (NPDB). Large magnitude $(M>7.0)$ historical thrust earthquakes have occurred repeatedly along the NPDB (Figure 1) [Plafker and Ward, 1992; Goes et al., 1993; Lundgren et al., 1993; Tajima and Kikuchi, 1995].

[13] Maximum uplift and shortening of the Costa Rican fore arc occurs directly inboard of Cocos Ridge. Quaternary shortening exceeds $15 \mathrm{~km}(10-$ $40 \mathrm{~mm} \mathrm{a}^{-1}$ ) across the fore arc Fila Costeña fold and thrust belt [Fisher et al., 2004; Sitchler et al., 2007]. The Cordillera de Talamanca, a roughly $4 \mathrm{~km}$ high mountain range, located between the Fila Costeña thrust belt and the NPDB (Figure 1) exposes plutonic rocks as young as $6 \mathrm{Ma}$ [MacMillan et al., 2004], implying rapid uplift. The Central Costa Rican Deformed Belt [Marshall et al., 2000] and faults of the Azuero Peninsula, Panama [Mann and Corrigan, 1990; Silver et al., 1990] cut the Central American isthmus northwest and southeast of Cocos Ridge, respectively, and mark the diffuse eastern and western boundaries of the Panama block.

\section{GPS Velocity Field}

[14] Our GPS velocity field is based on episodic (E) and continuous (C) GPS observations and resultant time series spanning the period 19932005. We combine EGPS data sets presented by Lundgren et al. [1993, 1999], Trenkamp et al. [2002], Norabuena et al. [2004], and Turner et al. [2007] with new EGPS and CGPS data to define the secular velocity field for this time period. All data were reprocessed in a consistent reference frame (ITRF00) (Table 1) using GIPSY-OASIS II [Zumberge et al., 1997] following the methods of Sella et al. [2002]. The networks within each country are variable in spatial and temporal sampling 


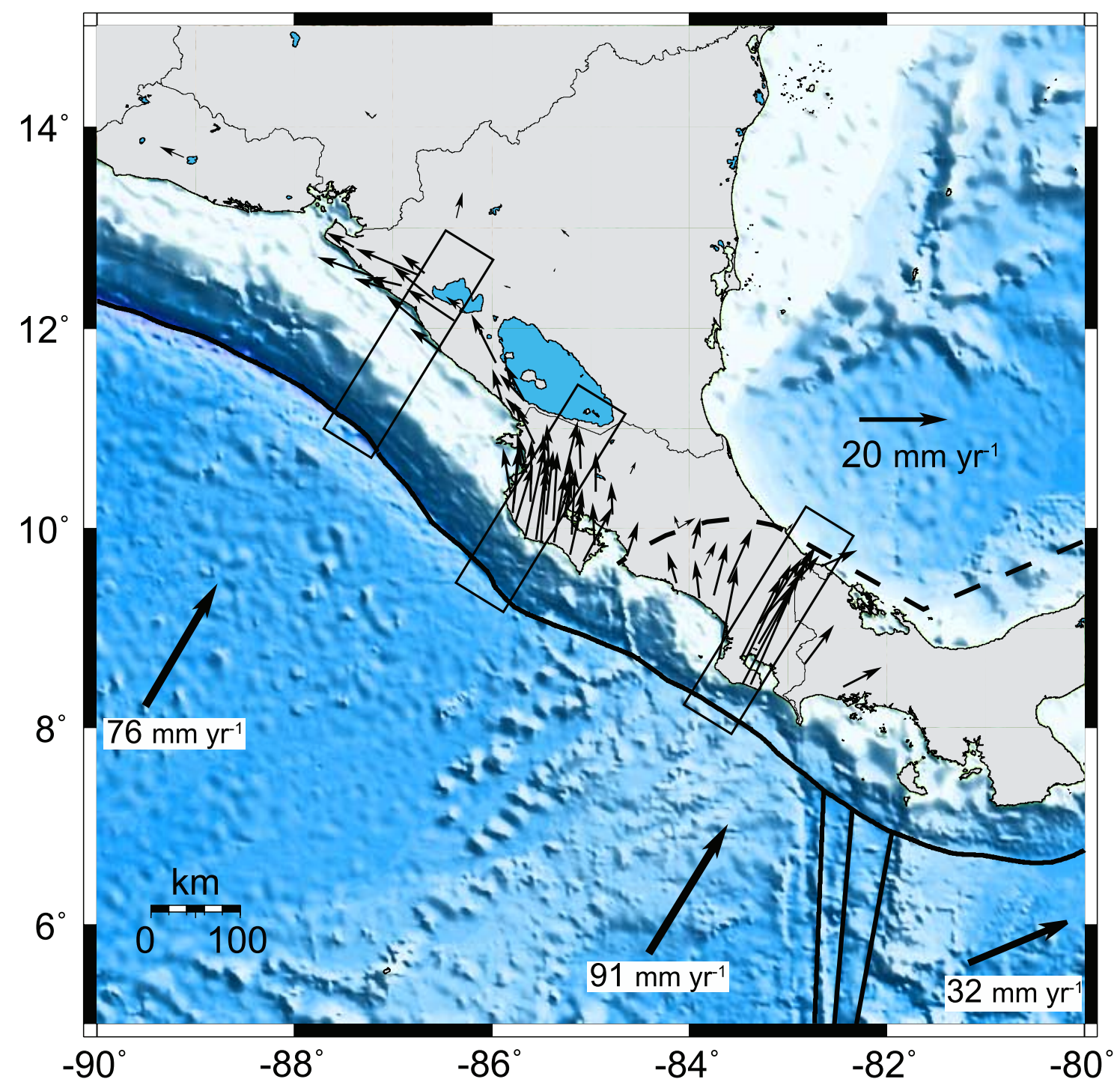

Figure 4. GPS-derived velocity field (ITRF-00) for Central America relative to a stable Caribbean plate [Lopez et al., 2006] (Table 2). Large black vectors indicate the relative rate and azimuth between the Cocos and Caribbean plates [DeMets, 2001] and Nazca-Caribbean plates [Sella et al., 2002]. Short black arrow $\left(20 \mathrm{~mm} \mathrm{a}^{-1}\right)$ shows scale for velocity vectors. Black boxes indicate area of data used for profiles shown in Figure 5.

(Figure 4 and Table 1). We estimate site velocities for 79 sites (69 EGPS and 10 CGPS). The velocities for three EGPS sites located in eastern Panama are given in Tables 1 and 2, but these sites are located outside of the model area and are not presented in Figure 4.

[15] Site velocity vectors are plotted relative to a stable Caribbean plate reference frame (Figure 4 and Table 2) [Lopez et al., 2006]. The horizontal velocity components that are respectively parallel and perpendicular to the plate convergence direction are plotted for three margin-normal transects in Figure 5 (see Figure 4 for transect locations). Two features of the velocity field are noteworthy. First, rates of displacement are high in the outer fore arc; sites on Nicoya and Osa peninsulas move northeastward at rates up to 25 and $44 \mathrm{~mm} \mathrm{a}^{-1}$, respectively. Second, while the margin-parallel component is insignificant in southern Costa Rica (i.e., essentially all motion is parallel to the plate convergence direction), it is significant in the other two transects (Figure 5). Sites in a $\sim 50 \mathrm{~km}$ wide swath normal to the trench and centered on Cocos Ridge (offshore) and Osa Peninsula move essentially parallel to the Cocos-Caribbean relative convergence direction $\left(\sim \mathrm{N} 21^{\circ} \mathrm{E}\right)$. Northwest and southeast of Osa Peninsula, velocities are rotated counterclockwise and clockwise, respectively, from the plate convergence direction (Figure 4). 
Table 1. GPS Velocities Relative to ITRF-00 and Weighted RMS Scatter

\begin{tabular}{|c|c|c|c|c|c|c|c|c|c|c|}
\hline \multirow[b]{2}{*}{ Site } & \multirow[b]{2}{*}{ Latitude } & \multirow[b]{2}{*}{ Longitude } & \multirow[b]{2}{*}{$\Delta T$} & \multirow[b]{2}{*}{$N$} & \multicolumn{3}{|c|}{$\begin{array}{l}\text { Velocity } \\
\left(\mathrm{mm} \mathrm{a}^{-1}\right)\end{array}$} & \multicolumn{3}{|c|}{$\begin{array}{c}\text { WRMS } \\
(\mathrm{mm})\end{array}$} \\
\hline & & & & & North & East & Vertical & North & East & Vertical \\
\hline ACOS & 10.54 & -84.60 & 9.04 & 57 & $9.3 \pm 0.8$ & $14.4 \pm 1.2$ & $-1.5 \pm 2.0$ & 5.6 & 9.8 & 15.6 \\
\hline AGUJ & 9.72 & -84.62 & 9.04 & 16 & $16.2 \pm 0.8$ & $16.5 \pm 1.5$ & $-0.3 \pm 2.1$ & 4.6 & 9.6 & 12.9 \\
\hline ALEX & 8.43 & -83.37 & 3.03 & 8 & $46.8 \pm 2.1$ & $31.6 \pm 1.9$ & $-8.4 \pm 6.8$ & 3.9 & 3.9 & 13.2 \\
\hline ANA1 & 12.08 & -86.37 & 1.98 & 20 & $14.6 \pm 3.2$ & $-2.4 \pm 4.9$ & $-5.7 \pm 8.2$ & 4.8 & 8.2 & 13.1 \\
\hline BAGA & 10.54 & -85.26 & 2.98 & 8 & $21.9 \pm 1.8$ & $16.6 \pm 6.5$ & $1.4 \pm 4.7$ & 3.2 & 12.6 & 7.9 \\
\hline BAHA $^{\mathrm{a}}$ & 9.05 & -79.52 & 2.04 & 5 & $-11.0 \pm 5.3$ & $-21.9 \pm 8.0$ & $28.9 \pm 13.0$ & 6.2 & 9.7 & 16.0 \\
\hline BALL & 10.38 & -85.44 & 9.03 & 14 & $22.7 \pm 1.1$ & $11.8 \pm 0.9$ & $-1.2 \pm 2.0$ & 6.1 & 5.7 & 11.1 \\
\hline $\mathrm{BONG}$ & 9.74 & -85.20 & 3.00 & 8 & $22.5 \pm 1.8$ & $16.9 \pm 4.1$ & $-0.5 \pm 6.3$ & 3.2 & 8.1 & 11.9 \\
\hline BRAT & 9.55 & -82.89 & 6.10 & 12 & $14.6 \pm 1.1$ & $18.3 \pm 3.1$ & $-8.6 \pm 3.6$ & 4.3 & 13.1 & 15.0 \\
\hline CABU & 10.13 & -84.77 & 9.24 & 18 & $15.5 \pm 1.1$ & $12.5 \pm 1.5$ & $3.3 \pm 3.2$ & 6.8 & 9.9 & 22.8 \\
\hline CAMP & 8.63 & -82.83 & 9.05 & 15 & $18.1 \pm 1.2$ & $22.4 \pm 0.7$ & $-2.5 \pm 2.2$ & 6.7 & 4.8 & 13.2 \\
\hline CANA & 9.45 & -83.6 & 3.02 & 8 & $22.3 \pm 2.0$ & $20.5 \pm 3.8$ & $-2.9 \pm 7.9$ & 3.7 & 7.6 & 15.9 \\
\hline CARA & 8.44 & -83.46 & 9.95 & 20 & $35.8 \pm 2.0$ & $29.0 \pm 1.3$ & $-3.5 \pm 3.1$ & 13.5 & 9.4 & 24.3 \\
\hline CEBA & 10.24 & -85.77 & 3.03 & 8 & $22.6 \pm 1.5$ & $14.6 \pm 2.4$ & $-0.1 \pm 5.5$ & 2.7 & 4.9 & 10.1 \\
\hline CORI & 12.51 & -87.19 & 1.99 & 54 & $10.6 \pm 1.8$ & $-5.2 \pm 2.6$ & $-0.3 \pm 5.7$ & 3.1 & 5.3 & 9.7 \\
\hline CORN & 12.17 & -83.06 & 2.11 & 10 & $6.3 \pm 2.4$ & $11.0 \pm 3.8$ & $-8.8 \pm 7.9$ & 3.4 & 5.9 & 11.1 \\
\hline CORO & 9.86 & -85.36 & 3.00 & 8 & $26.6 \pm 2.0$ & $16.5 \pm 4.5$ & $-3.1 \pm 6.7$ & 3.7 & 9.0 & 12.8 \\
\hline CRIS & 8.40 & -82.44 & 2.05 & 6 & $12.4 \pm 3.7$ & $25.4 \pm 10.9$ & $14.6 \pm 22.6$ & 4.5 & 13.9 & 31.0 \\
\hline CRUZ & 11.05 & -85.63 & 8.29 & 19 & $12.6 \pm 1.1$ & $6.0 \pm 1.3$ & $-4.8 \pm 2.4$ & 5.9 & 7.7 & 14.3 \\
\hline DIRI & 10.27 & -85.61 & 3.03 & 10 & $24.2 \pm 2.1$ & $13.1 \pm 4.0$ & $6.3 \pm 6.2$ & 4.1 & 8.5 & 12.6 \\
\hline ELBQ & 11.27 & -85.66 & 3.84 & 20 & $13.4 \pm 2.9$ & $4.8 \pm 2.4$ & $2.3 \pm 3.4$ & 8.1 & 7.3 & 8.5 \\
\hline ELCO & 12.8 .0 & -87.4 .0 & 2.53 & 17 & $9.1 \pm 2.4$ & $3.1 \pm 3.7$ & $2.1 \pm 5.0$ & 4.3 & 7.5 & 8.2 \\
\hline $\mathrm{ESTI}^{\mathrm{b}}$ & 13.09 & -86.36 & 1.15 & 403 & $15.1 \pm 2.6$ & $-24.4 \pm 5.5$ & $36.0 \pm 8.4$ & 3.6 & 8.9 & 12.3 \\
\hline ESTR & 9.79 & -83.96 & 3.02 & 7 & $15.2 \pm 1.9$ & $15.2 \pm 2.6$ & $-3.2 \pm 10.6$ & 3.4 & 5.1 & 21.3 \\
\hline ETCG & 9.99 & -84.1 & 6.25 & 24 & $9.8 \pm 1.1$ & $11.5 \pm 2.2$ & $3.9 \pm 3.2$ & 5.0 & 11.0 & 15.6 \\
\hline FLAM $^{\mathrm{a}}$ & 8.90 & -79.52 & 1.98 & 33 & $13.3 \pm 1.7$ & $9.5 \pm 4.0$ & $1.3 \pm 8.6$ & 2.7 & 7.3 & 15.8 \\
\hline GRAN & 10.56 & -85.65 & 10.25 & 24 & $17.4 \pm 0.6$ & $9.7 \pm 0.5$ & $1.1 \pm 1.6$ & 4.2 & 3.7 & 10.9 \\
\hline GUAR & 10.14 & -85.44 & 9.05 & 12 & $24.8 \pm 0.7$ & $15.0 \pm 1.0$ & $-5.8 \pm 1.9$ & 3.6 & 6.1 & 10.3 \\
\hline GUAT $^{\mathrm{b}}$ & 14.59 & -90.52 & 2.53 & 833 & $1.5 \pm 1.2$ & $4.0 \pm 1.7$ & $1.6 \pm 3.3$ & 3.8 & 6.1 & 9.6 \\
\hline GUIO & 9.92 & -85.65 & 2.99 & 8 & $32.7 \pm 2.2$ & $18.9 \pm 2.3$ & $-6.4 \pm 5.4$ & 4.0 & 4.8 & 9.6 \\
\hline HATI & 9.30 & -83.90 & 3.01 & 6 & $17.5 \pm 2.9$ & $11.9 \pm 2.2$ & $1.1 \pm 5.3$ & 5.0 & 4.3 & 8.8 \\
\hline HELE & 9.06 & -83.10 & 2.98 & 8 & $24.5 \pm 1.9$ & $21.9 \pm 1.7$ & $0.5 \pm 6.4$ & 3.5 & 3.6 & 12.1 \\
\hline HOJA & 10.07 & -85.38 & 3.03 & 8 & $26.2 \pm 0.8$ & $14.1 \pm 3.6$ & $1.3 \pm 3.7$ & 1.3 & 7.2 & 5.3 \\
\hline $\mathrm{HUA}^{\mathrm{b}}$ & 10.01 & -85.35 & 2.15 & 509 & $21.7 \pm 1.1$ & $18.0 \pm 1.6$ & $0.8 \pm 3.8$ & 2.8 & 4.8 & 8.5 \\
\hline INDI $^{\mathrm{b}}$ & 9.86 & -85.50 & 2.32 & 824 & $26.6 \pm 1.3$ & $14.9 \pm 1.6$ & $-7.1 \pm 3.8$ & 3.6 & 5.4 & 10.6 \\
\hline JICA & 9.97 & -85.13 & 8.99 & 19 & $18.3 \pm 1.3$ & $14.6 \pm 1.5$ & $-2.5 \pm 1.9$ & 7.5 & 10.1 & 11.4 \\
\hline LENI & 12.42 & -86.90 & 4.49 & 76 & $7.3 \pm 1.3$ & $1.2 \pm 1.2$ & $3.2 \pm 2.8$ & 5.0 & 5.1 & 10.8 \\
\hline LEON & 9.93 & -85.18 & 2.96 & 6 & $25.3 \pm 2.8$ & $14.0 \pm 2.9$ & $0.8 \pm 4.7$ & 4.9 & 5.4 & 7.1 \\
\hline LIBE & 10.65 & -85.42 & 3.24 & 20 & $17.5 \pm 2.5$ & $12.0 \pm 3.3$ & $-3.8 \pm 6.0$ & 6.0 & 8.7 & 15.7 \\
\hline LIMO & 9.96 & -83.02 & 2.96 & 7 & $9.0 \pm 2.4$ & $15.4 \pm 4.0$ & $-6.7 \pm 4.3$ & 4.2 & 7.7 & 6.4 \\
\hline LOCA & 9.67 & -85.07 & 3.02 & 8 & $22.1 \pm 2.8$ & $21.9 \pm 2.4$ & $2.7 \pm 5.9$ & 5.2 & 4.9 & 11.1 \\
\hline MANA $^{\mathrm{b}}$ & 12.14 & -86.24 & 1.54 & 485 & $6.4 \pm 1.9$ & $4.1 \pm 2.5$ & $2.9 \pm 5.4$ & 3.4 & 5.4 & 9.2 \\
\hline MARE & 8.68 & -83.70 & 3.09 & 21 & $34.2 \pm 2.6$ & $19.0 \pm 3.0$ & $-8.9 \pm 6.6$ & 6.0 & 7.7 & 17.0 \\
\hline MATA & 10.35 & -85.81 & 10.25 & 22 & $18.8 \pm 0.9$ & $10.5 \pm 1.0$ & $0.0 \pm 1.7$ & 6.2 & 7.5 & 11.7 \\
\hline MNAZ & 9.61 & -82.67 & 2.01 & 9 & $11.2 \pm 3.4$ & $24.2 \pm 6.1$ & $-21.1 \pm 12.2$ & 4.5 & 8.6 & 17.5 \\
\hline MORA & 8.62 & -83.45 & 3.01 & 7 & $36.6 \pm 2.4$ & $28.0 \pm 5.9$ & $-13.7 \pm 5.7$ & 4.3 & 11.3 & 10.0 \\
\hline $\mathrm{OCHO}$ & 11.66 & -85.95 & 2.51 & 11 & $20 \pm 3.0$ & $4.7 \pm 6.1$ & $5.1 \pm 7.9$ & 5.1 & 11.1 & 14.0 \\
\hline $\mathrm{OVSI}^{\mathrm{b}}$ & 10.00 & -84.11 & 1.97 & 577 & $8.9 \pm 2.0$ & $18.1 \pm 2.5$ & $3.4 \pm 4.9$ & 4.6 & 7.0 & 12.0 \\
\hline PALO & 10.24 & -85.22 & 2.96 & 6 & $19.7 \pm 1.4$ & $13.2 \pm 0.7$ & $0.1 \pm 4.7$ & 2.4 & 1.5 & 7.1 \\
\hline PAQU & 9.83 & -84.95 & 9.02 & 18 & $18.5 \pm 1.1$ & $12.4 \pm 1.6$ & $0.0 \pm 2.4$ & 6.4 & 10.2 & 15.5 \\
\hline PAZC & 12.29 & -86.59 & 2.53 & 19 & $15.2 \pm 3.7$ & $-0.7 \pm 2.1$ & $4.8 \pm 6.5$ & 6.9 & 4.5 & 12.7 \\
\hline $\mathrm{PIST}^{\mathrm{a}}$ & 7.98 & -80.40 & 2.00 & 4 & $7.4 \pm 4.6$ & $1.1 \pm 4.8$ & $-10.4 \pm 9.5$ & 5.0 & 5.5 & 10.1 \\
\hline PLMA & 9.32 & -83.74 & 3.02 & 9 & $21.8 \pm 2.4$ & $17.3 \pm 3.5$ & $-3.8 \pm 6.0$ & 4.5 & 7.2 & 11.6 \\
\hline $\mathrm{POCH}$ & 11.77 & -86.50 & 4.24 & 27 & $2.8 \pm 3.2$ & $-0.9 \pm 1.8$ & $1.3 \pm 3.3$ & 10.2 & 6.4 & 10.1 \\
\hline PONE & 12.38 & -87.02 & 2.10 & 18 & $8.5 \pm 3.1$ & $0.5 \pm 2.2$ & $3.1 \pm 5.9$ & 4.9 & 4.0 & 8.4 \\
\hline PORT & 12.57 & -85.36 & 2.54 & 14 & $6.4 \pm 2.7$ & $12.3 \pm 3.7$ & $3.6 \pm 5.8$ & 4.8 & 7.3 & 10.0 \\
\hline POTR & 10.84 & -85.56 & 4.23 & 11 & $17.8 \pm 2.0$ & $6.5 \pm 4.4$ & $10.2 \pm 5.7$ & 5.4 & 12.7 & 16.9 \\
\hline PUEC & 14.04 & -83.38 & 2.09 & 9 & $4.8 \pm 3.1$ & $10.8 \pm 3.4$ & $-2.2 \pm 8$ & 4.2 & 5.1 & 10.9 \\
\hline PUJE $^{b}$ & 10.11 & -85.27 & 2.06 & 695 & $16.9 \pm 1.5$ & $15.1 \pm 1.7$ & $1.1 \pm 3.8$ & 3.8 & 5.1 & 8.6 \\
\hline
\end{tabular}


Table 1. (continued)

\begin{tabular}{|c|c|c|c|c|c|c|c|c|c|c|}
\hline \multirow[b]{2}{*}{ Site } & \multirow[b]{2}{*}{ Latitude } & \multirow[b]{2}{*}{ Longitude } & \multirow[b]{2}{*}{$\Delta T$} & \multirow[b]{2}{*}{$N$} & \multicolumn{3}{|c|}{$\begin{array}{l}\text { Velocity } \\
\left(\mathrm{mm} \mathrm{a}^{-1}\right)\end{array}$} & \multicolumn{3}{|c|}{$\begin{array}{l}\text { WRMS } \\
(\mathrm{mm})\end{array}$} \\
\hline & & & & & North & East & Vertical & North & East & Vertical \\
\hline RINC & 8.69 & -83.48 & 3.01 & 7 & $30.9 \pm 2.9$ & $25.8 \pm 3.8$ & $-11.6 \pm 4.2$ & 5.2 & 7.3 & 6.3 \\
\hline RIOB & 12.92 & -85.22 & 2.55 & 14 & $7.7 \pm 1.8$ & $9.2 \pm 6.1$ & $5.2 \pm 5.6$ & 3.1 & 11.8 & 9.5 \\
\hline ROTA & 12.52 & -86.72 & 1.08 & 7 & $13.0 \pm 4.2$ & $-7.6 \pm 5.1$ & $12.3 \pm 11.8$ & 2.9 & 3.9 & 7.5 \\
\hline SAMA & 9.88 & -85.54 & 9.03 & 19 & $30.5 \pm 1.1$ & $16.4 \pm 1.1$ & $-15.1 \pm 2.9$ & 6.8 & 7.2 & 20.3 \\
\hline SANA & 12.52 & -81.73 & 9.16 & 33 & $6.7 \pm 0.6$ & $12.2 \pm 0.9$ & $-1.5 \pm 2.4$ & 3.6 & 6.5 & 18.3 \\
\hline SIEM & 9.44 & -84.13 & 3.02 & 8 & $15.3 \pm 2.7$ & $10.4 \pm 4.7$ & $4.0 \pm 5.4$ & 5.1 & 9.4 & 9.8 \\
\hline SJOS & 10.36 & -84.94 & 9.24 & 17 & $17.6 \pm 1.0$ & $12.8 \pm 1.3$ & $0.3 \pm 3.6$ & 6.2 & 8.6 & 26 \\
\hline SJUA & 10.06 & -85.75 & 9.02 & 17 & $22.8 \pm 1.3$ & $18.2 \pm 1.5$ & $-7.3 \pm 2.0$ & 7.9 & 9.5 & 12.3 \\
\hline $\mathrm{SLOR}^{\mathrm{b}}$ & 13.42 & -87.43 & 0.59 & 194 & $-2.5 \pm 4.2$ & $7.3 \pm 8.0$ & $-8.7 \pm 15.3$ & 2.9 & 6.6 & 10.8 \\
\hline SSIA $^{\mathrm{b}}$ & 13.69 & -89.11 & 0.99 & 307 & $-1.4 \pm 3.4$ & $1.0 \pm 6.2$ & $-4.7 \pm 9.0$ & 4.0 & 8.5 & 10.4 \\
\hline TEGU $^{\mathrm{b}}$ & 14.09 & -87.21 & 1.73 & 569 & $6.1 \pm 1.6$ & $12.6 \pm 2.7$ & $-7.3 \pm 5.3$ & 3.1 & 6.2 & 11.3 \\
\hline TENO & 10.60 & -85.10 & 3.03 & 6 & $17.9 \pm 4.3$ & $12.2 \pm 4.8$ & $-16.7 \pm 5.4$ & 7.7 & 8.8 & 8.9 \\
\hline TEUS & 12.40 & -85.81 & 2.55 & 16 & $5.8 \pm 2.1$ & $9.8 \pm 2.8$ & $-5.5 \pm 7.7$ & 3.9 & 5.8 & 15.5 \\
\hline TIG2 & 9.04 & -83.29 & 2.98 & 7 & $25.8 \pm 1.6$ & $26.2 \pm 4.7$ & $-8.5 \pm 5.2$ & 2.8 & 8.9 & 8.8 \\
\hline TRAN & 12.02 & -86.68 & 2.07 & 9 & $14.4 \pm 2.7$ & $0.7 \pm 3.8$ & $-4.3 \pm 7.2$ & 3.6 & 5.6 & 9.3 \\
\hline VENA & 10.16 & -85.79 & 2.99 & 9 & $25.0 \pm 2.2$ & $14.6 \pm 4.5$ & $-1.0 \pm 5.9$ & 4.1 & 9.1 & 11.3 \\
\hline VINC & 11.29 & -85.89 & 4.03 & 102 & $14.3 \pm 1.6$ & $-1.3 \pm 2.4$ & $-0.3 \pm 3.2$ & 6.0 & 10.0 & 12.0 \\
\hline VNEC & 8.83 & -83.29 & 2.99 & 8 & $28.0 \pm 2.8$ & $26.4 \pm 3.9$ & $4.6 \pm 7.8$ & 5.3 & 7.8 & 15.6 \\
\hline VUEL & 9.62 & -83.85 & 9.03 & 15 & $13.1 \pm 1.7$ & $16.7 \pm 1.4$ & $6.0 \pm 2.9$ & 9.8 & 8.9 & 19.2 \\
\hline ZUMA & 9.65 & -85.08 & 1.97 & 7 & $14.4 \pm 3.2$ & $8.6 \pm 4.9$ & $-17.2 \pm 8.7$ & 3.9 & 6.4 & 10.6 \\
\hline
\end{tabular}

${ }^{\mathrm{a}}$ Eastern Panama sites. These sites were not used in the study.

${ }^{\mathrm{b}}$ CGPS sites.

The velocity field is poorly constrained in northern Panama, but the available data suggest clockwise rotation away from Cocos Ridge. In Nicaragua, counterclockwise rotation from the plate convergence direction is extreme, with many sites moving essentially parallel to the trench [Turner et al., 2007].

[16] Recent analysis of time series for three CGPS sites on Nicoya Peninsula [Protti et al., 2004] and pressure and temperature records [Davis and Villinger, 2006] offshore Nicoya Peninsula suggest the occurrence of slow slip transients on this portion of the Central American margin. The limited temporal and spatial extents of CGPS data at this time make it difficult to constrain the effects of this process on EGPS velocities [e.g., Larson et al., 2004].

\section{Modeling}

[17] The interseismic velocity field in Central America and in most other subduction plate boundaries is usually interpreted in terms of two major processes: strain accumulation due to locking on the shallow (less than $50 \mathrm{~km}$ depth) part of the plate boundary, and trench-parallel translation of the fore arc due to oblique convergence and slip partitioning [e.g., Dixon, 1993; Lundgren et al., 1999; McCaffrey, 2002; Norabuena et al., 2004; Turner et al., 2007]. To a first approximation, the former may be regarded as a purely elastic (earthquake cycle) process, generating no net deformation of overriding plate lithosphere, while the latter is capable of generating considerable long-term displacement of fore-arc terrains [Beck, 1991]. In the first part of this paper, we investigate the surface kinematics of the region using block models, inverting for interseismic elastic strain accumulation (fault coupling) on block bounding faults and fore-arc block rotation (slip partitioning). We show that oblique convergence and mechanical coupling between the subducting and overriding plates do not fully explain the pattern of trench-parallel motion we observe. In the second part of the paper, we focus on longer time scales, and test whether a mechanical model of Cocos Ridge collision can reproduce our observed surface velocity field.

\subsection{Interseismic Elastic Strain Accumulation Models}

[18] For most of the region, three-dimensional models are clearly required since there is significant motion perpendicular to the plate convergence direction (Figures 4 and 5). However, in southern Costa Rica, where motion is essentially parallel to the plate convergence direction, a two-dimensional elastic half-space model of a locked, dipping thrust fault [Savage, 1983] adequately represents the data. Figure 6 shows the fit of our GPS velocities to this 
Table 2. GPS Velocities Relative to Stable Caribbean Plate Euler Vector of Lopez et al. [2006]

\begin{tabular}{|c|c|c|c|c|}
\hline \multirow[b]{2}{*}{ Site } & \multirow[b]{2}{*}{ Latitude } & \multirow[b]{2}{*}{ Longitude } & \multicolumn{2}{|c|}{$\begin{array}{l}\text { Velocity } \\
\left(\mathrm{mm} \mathrm{a}^{-1}\right)\end{array}$} \\
\hline & & & North & East \\
\hline $\mathrm{COS}$ & 10.55 & -84.60 & $3.84 \pm 0.9$ & $1.75 \pm 1.24$ \\
\hline AGUJ & 9.72 & -84.63 & $10.75 \pm 0.9$ & $3.57 \pm 1.53$ \\
\hline ALEX & 8.43 & -83.38 & $40.97 \pm 1.93$ & $18.21 \pm 2.14$ \\
\hline ANA1 & 12.08 & -86.38 & $9.68 \pm 3.23$ & $-14.48 \pm 4.91$ \\
\hline BAGA & 10.54 & -85.26 & $16.64 \pm 1.85$ & $3.96 \pm 6.51$ \\
\hline BAHA $^{\mathrm{a}}$ & 9.06 & -79.52 & $-17.98 \pm 5.31$ & $-35.15 \pm 8.01$ \\
\hline BALL & 10.38 & -85.45 & $17.5 \pm 0.95$ & $-0.89 \pm 1.19$ \\
\hline BONG & 9.74 & & $17.22 \pm 1.85$ & $3.98 \pm 4.11$ \\
\hline BRAT & 9.55 & -82.89 & $8.62 \pm 1.17$ & $5.28 \pm 3.12$ \\
\hline CABU & 10.14 & -84.78 & $10.09 \pm 1.18$ & $-0.29 \pm 1.53$ \\
\hline CAMP & 8.64 & -82.83 & $12.1 \pm 0.77$ & $9.07 \pm 1.26$ \\
\hline CANA & 9.46 & -83.61 & $16.54 \pm 2.04$ & $7.46 \pm 3.81$ \\
\hline CARA & 8.44 & -83.46 & $29.99 \pm 1.34$ & $15.61 \pm 2.04$ \\
\hline CEBA & 10.25 & -85 & $17.5 \pm 1.56$ & $1.87 \pm 2$ \\
\hline CORI & 12.52 & -87 & $5.94 \pm 1.86$ & $-17.11 \pm 2.62$ \\
\hline CORN & 12.18 & -83.06 & $0.38 \pm 2.43$ & $-1.11 \pm 3.81$ \\
\hline CORO & 9.86 & -85.37 & $21.37 \pm 2.05$ & $3.63 \pm 4.51$ \\
\hline CRIS & 8.41 & -82 & $6.29 \pm 3.72$ & $11.99 \pm$ \\
\hline CRUZ & 11.05 & -85 & $8.05 \pm 1.09$ & $-7.05 \pm$ \\
\hline DIRI & 10.27 & 1 & $19.05 \pm 2.15$ & $0.37 \pm$ \\
\hline ELBQ & 11.28 & -85.67 & $8.26 \pm 2.32$ & $-7.57 \pm 2.93$ \\
\hline ELCO & 12.81 & -87.40 & $4.5 \pm 2.45$ & $-8.70 \pm 3.71$ \\
\hline $\mathrm{ESTI}^{\mathrm{b}}$ & 13.10 & -86.36 & $7.98 \pm 1.10$ & $1.79 \pm 2.02$ \\
\hline ESTR & 9.80 & -83 & $9.54 \pm 1.94$ & $2.28 \pm 2.62$ \\
\hline ETCG & 10.00 & -84.11 & $4.19 \pm 1.17$ & $-1.35 \pm 2.22$ \\
\hline FLAM $^{\mathrm{a}}$ & 8.91 & -79.52 & $6.32 \pm 1.73$ & $-3.80 \pm 4.01$ \\
\hline GRAN & 10.56 & -85 & $12.26 \pm 0.57$ & $-2.92 \pm 0.75$ \\
\hline GUAR & 10.14 & -85.45 & $19.60 \pm 0.82$ & $2.23 \pm 1.05$ \\
\hline GUAT $^{\mathrm{b}}$ & 14.59 & -90.52 & $-2.24 \pm 0.88$ & $-6.3 \pm 1.04$ \\
\hline UIO & & -85 & $27.56 \pm 2.24$ & $6.05 \pm$ \\
\hline HATI & 9.30 & -83 & $11.83 \pm 2.22$ & $-1.19 \pm$ \\
\hline HELE & 9.06 & -83 & $9 \pm 1.73$ & $8.72 \pm 1.94$ \\
\hline HOJA & 10.08 & -85.38 & $20.98 \pm 0.91$ & $1.30 \pm 3.61$ \\
\hline HUA2 ${ }^{\mathrm{b}}$ & 10.02 & -85.35 & $16.47 \pm 1.18$ & $5.18 \pm 1.63$ \\
\hline INDI $^{\mathrm{b}}$ & 9.86 & -85.50 & $23.01 \pm 0.62$ & $1.63 \pm 0.72$ \\
\hline JICA & 9.98 & -85.14 & $13.00 \pm 1.37$ & $1.76 \pm 1.53$ \\
\hline LENI & 12.43 & -86.91 & $2.55 \pm 1.23$ & $-10.74 \pm 1.38$ \\
\hline LEON & 9.94 & -85.19 & $20.02 \pm 2.83$ & $1.15 \pm 2.92$ \\
\hline LIBE & 10.65 & -85 & $12.29 \pm 2.54$ & $-0.60 \pm 3$ \\
\hline $\mathrm{O}$ & & -83 & $3.06 \pm 2.43$ & $2.52 \pm 4.01$ \\
\hline LOCA & 9.67 & -85.07 & $16.78 \pm 2.42$ & $8.96 \pm 2.83$ \\
\hline MANA $^{\mathrm{b}}$ & & & & $-6.55 \pm 1.04$ \\
\hline MARE & 8.69 & -83 & $28.47 \pm 2.63$ & $5.70 \pm 3.02$ \\
\hline MATA & 10.36 & -85.81 & $13.71 \pm 0.99$ & $-2.19 \pm 1.06$ \\
\hline MNAZ & 9.62 & -82.67 & $5.16 \pm 3.42$ & $11.20 \pm 6.11$ \\
\hline MORA & 8.63 & -83.45 & $30.79 \pm 2.43$ & $14.67 \pm 5.91$ \\
\hline $\mathrm{OCHO}$ & 11.66 & -85.96 & $14.95 \pm 3.03$ & $-7.53 \pm 6.11$ \\
\hline $\mathrm{OVSI}^{\mathrm{b}}$ & 10.00 & -84.11 & $3.29 \pm 2.04$ & $5.25 \pm 2.52$ \\
\hline PALO & 10.24 & -85.22 & $14.43 \pm 0.76$ & $0.46 \pm 1.47$ \\
\hline PAQU & & -84.96 & $13.15 \pm 1.18$ & $-0.49 \pm 1.63$ \\
\hline PAZC & 12.29 & -86.59 & $10.35 \pm 2.12$ & $-12.7 \pm 3.73$ \\
\hline & & -80.41 & $0.68 \pm 4.61$ & $-12.49 \pm 4.81$ \\
\hline PLMA & 9.33 & -83.75 & $16.08 \pm 2.43$ & $4.22 \pm 3.51$ \\
\hline $\mathrm{POCH}$ & 11.77 & -86.51 & $7.22 \pm 1.82$ & $-9.58 \pm 3.23$ \\
\hline PONE & 12.38 & -87.02 & $3.78 \pm 2.22$ & $-11.46 \pm 3.14$ \\
\hline PORT & 12.57 & -85.37 & $1.17 \pm 2.73$ & $0.38 \pm 3.71$ \\
\hline $\mathrm{PO}^{-}$ & 10.85 & -85.57 & $12.63 \pm 2.05$ & $-6.03 \pm 4.41$ \\
\hline PUEC & 14.04 & -83.38 & $-1.03 \pm 3.13$ & $-0.64 \pm 3.41$ \\
\hline
\end{tabular}

Table 2. (continued)

\begin{tabular}{|c|c|c|c|c|}
\hline \multirow[b]{2}{*}{ Site } & \multirow[b]{2}{*}{ Latitude } & \multirow[b]{2}{*}{ Longitude } & \multicolumn{2}{|c|}{$\begin{array}{l}\text { Velocity } \\
\left(\mathrm{mm} \mathrm{a}^{-1}\right)\end{array}$} \\
\hline & & & North & East \\
\hline $\mathrm{PUJE}^{\mathrm{b}}$ & 10.11 & -85.27 & $11.64 \pm 1.56$ & $2.31 \pm 1.73$ \\
\hline RINC & 8.69 & -83.48 & $25.10 \pm 2.93$ & $12.50 \pm 3.81$ \\
\hline RIOB & 12.92 & -85.22 & $2.43 \pm 1.85$ & $-2.60 \pm 6.11$ \\
\hline ROTA & 12.53 & -86.73 & $8.19 \pm 4.23$ & $-19.51 \pm 5.11$ \\
\hline SAMA & 9.89 & -85.55 & $25.33 \pm 1.13$ & $3.54 \pm 1.20$ \\
\hline SANA & 12.52 & -81.73 & $0.38 \pm 0.70$ & $0.18 \pm 0.94$ \\
\hline SIEM & 9.45 & -84.13 & $9.70 \pm 2.73$ & $-2.63 \pm 4.71$ \\
\hline SJOS & 10.37 & -84.95 & $12.24 \pm 1.08$ & $0.09 \pm 1.34$ \\
\hline SJUA & 10.06 & -85.76 & $17.69 \pm 1.37$ & $5.40 \pm 1.53$ \\
\hline $\mathrm{SLOR}^{\mathrm{b}}$ & 13.42 & -87.44 & $-3.09 \pm 1.48$ & $-3.87 \pm 2.71$ \\
\hline SSIA $^{\mathrm{b}}$ & 13.70 & -89.12 & $3.13 \pm 1.21$ & $-7.55 \pm 1.82$ \\
\hline TEGU $^{\mathrm{b}}$ & 14.09 & -87.21 & $1.44 \pm 1.67$ & $1.26 \pm 2.71$ \\
\hline TENO & 10.60 & -85.10 & $12.59 \pm 4.32$ & $-0.42 \pm 4.81$ \\
\hline TEUS & 12.41 & -85.81 & $0.71 \pm 2.15$ & $-2.17 \pm 2.81$ \\
\hline TIG2 & 9.05 & -83.29 & $19.94 \pm 1.65$ & $13.01 \pm 4.71$ \\
\hline TRAN & 12.02 & -86.69 & $9.58 \pm 2.74$ & $-11.39 \pm 3.81$ \\
\hline VENA & 10.16 & -85.79 & $19.90 \pm 2.24$ & $1.84 \pm 4.51$ \\
\hline VINC & 11.29 & -85.90 & $9.43 \pm 2.83$ & $-2.76 \pm 2.92$ \\
\hline VNEC & 8.84 & -83.30 & $22.14 \pm 2.83$ & $13.14 \pm 3.91$ \\
\hline VUEL & 9.63 & -83.85 & $7.41 \pm 1.43$ & $3.72 \pm 1.75$ \\
\hline ZUMA & 9.66 & -85.08 & $9.09 \pm 3.23$ & $-4.35 \pm 4.91$ \\
\hline
\end{tabular}

simple model, with two locked thrust faults of opposite dip, accounting for deformation on the main plate boundary and NPDB, respectively. The data are well fit with rates of $65 \mathrm{~mm} \mathrm{a}^{-1}$ (plate boundary) and $25 \mathrm{~mm} \mathrm{a}^{-1}$ (NPDB). Summed motion on the two faults $\left(90 \mathrm{~mm} \mathrm{a}^{-1}\right)$ is essentially identical to the long-term geologic estimate for overall Cocos-Caribbean relative plate motion, $90.5 \mathrm{~mm} \mathrm{a}^{-1}$ at this location [DeMets, 2001]. In the three-dimensional block models (below), we use the long-term geologic estimate as a formal constraint.

[19] In subduction zones where oblique convergence and mechanical coupling have led to slip partitioning and formation of margin-parallel strike slip faults (e.g., Sumatra) or collision has lead to back arc thrusting on faults antithetic to the main plate boundary thrust (e.g., New Hebrides), the fore arc is detached from the main overriding plate and can be considered as a separate tectonic block. This type of plate boundary system can be treated as a three-plate problem to quantify elastic strain accumulation on block-bounding faults and relative motion between the fore-arc block, and subducting and overriding plates [McCaffrey, 2002]. Here, our three-plate system consists of the Cocos and Caribbean plates and Central American fore-arc block (Figure 7). 


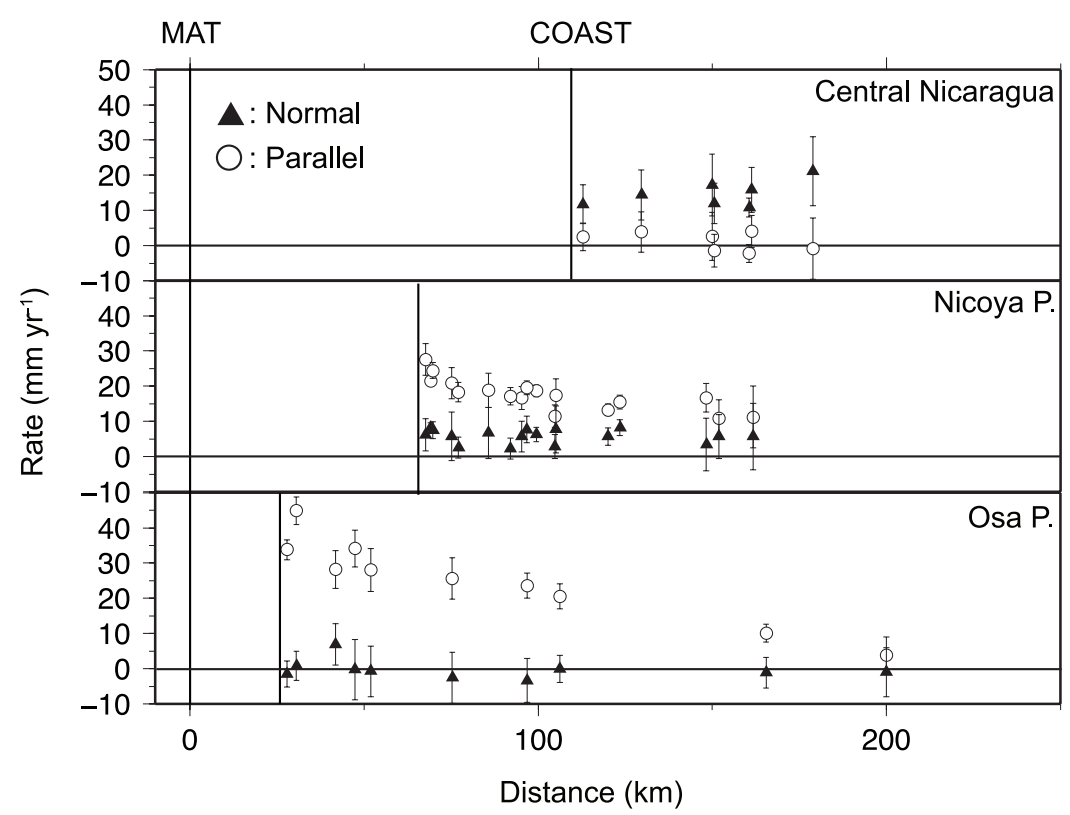

Figure 5. Velocity profiles for horizontal components parallel (open circles) and normal (black triangles) to CocosCaribbean relative plate motion vector of DeMets [2001] at the latitudes of (top) central Nicaragua, (middle) Nicoya Peninsula, and (bottom) Osa Peninsula. Normal component is absent at Osa Peninsula and between $\sim 5$ and $\sim 15 \mathrm{~mm} \mathrm{a}^{-1}$ from Nicoya Peninsula to central Nicaragua, indicating fore-arc sliver transport to the northwest.

[20] The geometry and location of the Central American seismogenic zone and NPDB are constrained using published geologic and geophysical data (Figure 7). The Central American seismogenic

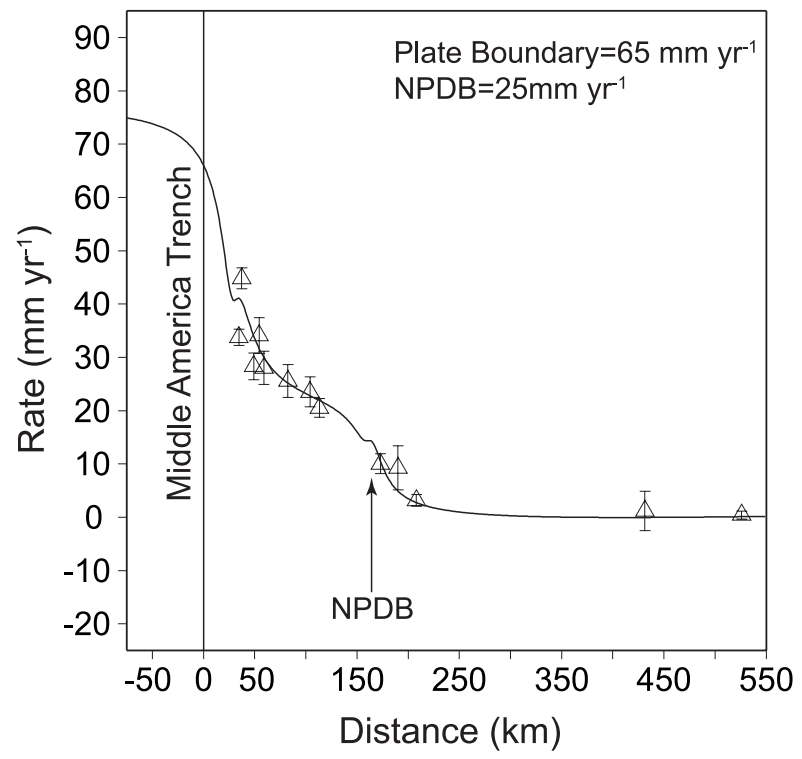

Figure 6. Two-dimensional elastic half-space model, following Savage [1983], for two antithetic thrust faults, the plate boundary thrust between the Cocos plate and fore arc and the North Panama Deformed Belt (NPDB). Data are along the transect at the latitude of Osa Peninsula (Figure 4). zone has variable dip and depth along strike in the model, matching available geophysical data [Adamek et al., 1987; Protti et al., 1994; Stavenhagen et al., 1996; Sallarès et al., 2000; DeShon et al., 2003, 2006; McIntosh et al., 2007]. We assume the NPDB

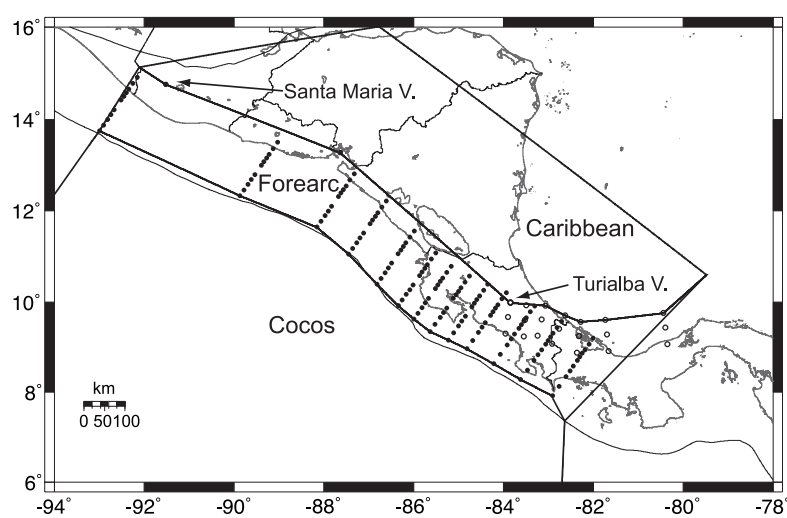

Figure 7. Map of the study area showing plate and block boundaries for the kinematic block model domain (thick black lines). We model this region as a three plate and block system: the Cocos and Caribbean plates and fore-arc block. Nodes used in modeling the magnitude and pattern of coupling are shown for the Central America seismogenic zone (black dots) and NPDB (open circles). Nodes are not visible for the fore-arc strike slip fault separating fore arc from Caribbean plate because of vertical orientation. Locations of Santa Maria and Turialba volcanoes are shown. 
has a constant dip based on the fault plane solution for the 1991 Valle de Estrella/El Limon earthquake [Goes et al., 1993; Suarez et al., 1995; Tajima and Kikuchi, 1995]. The fore-arc strike slip fault is modeled as a vertical fault following the strike of the Central American volcanic arc and roughly parallel to the margin (Figure 7). The fault begins at Turialba Volcano, Costa Rica, where it merges with the western extent of the NPDB, and ends at Santa Maria Volcano, Guatemala (Figure 7). The NPDB and fore-arc strike slip fault define the boundary between the fore arc and Caribbean plate.

[21] The Cocos-Caribbean angular velocity of DeMets [2001] and a fixed Caribbean plate reference frame are used as boundary conditions for the model. We simultaneously invert the horizontal and vertical components of the GPS velocity vectors (Figure 4 and Table 2) and earthquake slip vectors using DEFNODE [McCaffrey, 1992, 2002]. Earthquake slip vectors used in the inversion are filtered following McCaffrey [1992, 2002] and shown in Figure 2. We use only plate interface earthquakes from the global CMT database with $M \geq 6$ and depths less than $60 \mathrm{~km}$. We solve for (1) interseismic elastic strain accumulation and the magnitude and pattern of coupling on block bounding faults, (2) Euler vectors describing Central American fore-arc block motion relative to the Cocos and Caribbean plates, and (3) rates and azimuths of relative block motion using the new Euler vectors.

[22] The model fault planes are discretized in three dimensions by nodes spaced according to a resolution test (see below) and the fault plane geometries described above. Coupling $(\varphi)$ is defined as the ratio of "locked slip" to total relative block motion $(\varphi=0$ represents fault creep at the full relative plate rate, while $\varphi=1$ is equivalent to a fully locked fault, with a creep rate of zero). The magnitude of coupling can be constrained on individual nodes and across the fault plane [e.g., McCaffrey, 2002]. We test models where the magnitude and pattern of coupling are either defined (i.e., the distribution of coupling on the fault plane is prescribed) or not defined.

[23] Our data set has significant spatial variability, with closely spaced stations in northern and southern Costa Rica ( $25 \mathrm{~km}$ average station spacing), and much sparser sampling in central Costa Rica and Nicaragua (Figure 4). We test the resolving power of our geodetic network to estimate the degree and pattern of coupling along the seismogenic zone using "checkerboard tests." The fault

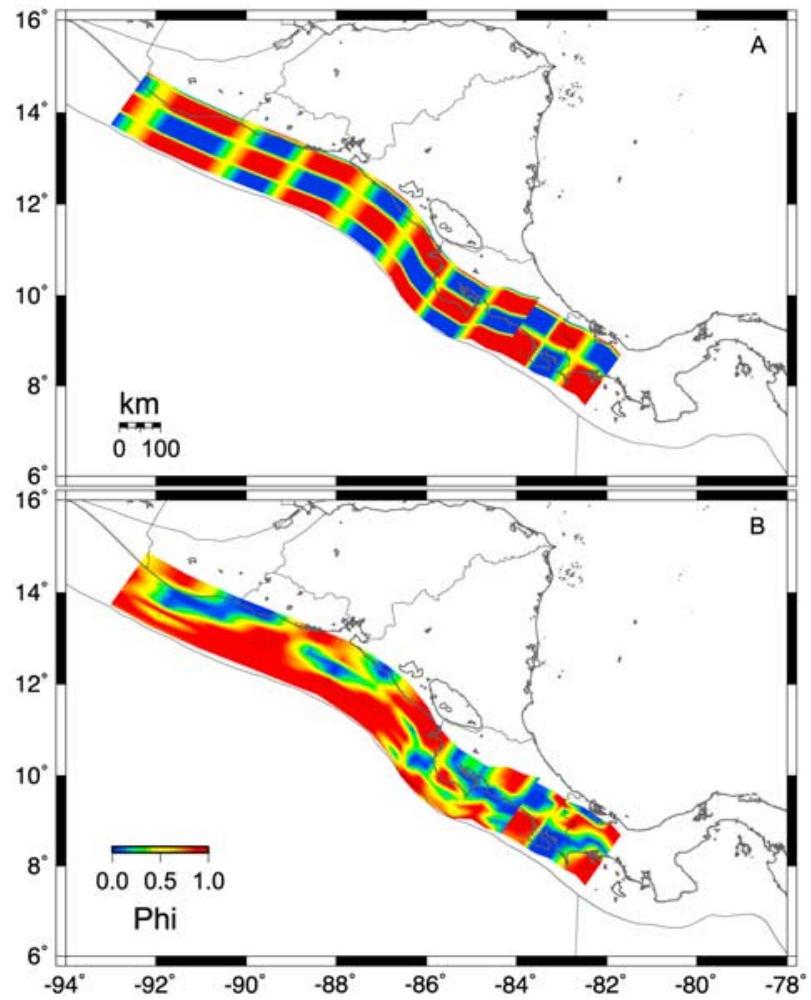

Figure 8. A resolution test for the pattern and amplitude of coupling along the Central America seismogenic zone utilizing the Central America GPS network. (a) Synthetic checkerboard input pattern of locking along the subduction interface. (b) Results from inverting the network surface velocities due to the synthetic locking pattern. The input pattern and amplitude are reasonably well recovered near Nicoya and Osa peninsulas. Recovery is poor offshore Nicaragua and northward (see text for discussion).

plane is discretized into rectangular patches of varying sizes, with either full $(\varphi=1.0)$ or no $(\varphi=0.0)$ coupling (Figure 8a). A forward model is then implemented to calculate the theoretical surface velocity at each site corresponding to the imposed slip pattern. The GPS measurement uncertainties are added to the synthetic velocity field and the resulting velocity field is then inverted for the degree and pattern of coupling. Figure $8 \mathrm{~b}$ shows the result of one such test (representing our final patch distribution), with patch size ranging from $75 \mathrm{~km}$ (along strike) and $\sim 50 \mathrm{~km}$ (downdip) in northern Costa Rica to as much as $500 \mathrm{~km}$ along strike in central Nicaragua and northward (Figure 8a). A test for the minimum patch size at the latitude of Nicoya peninsula indicates that patches with dimensions of $\sim 50 \mathrm{~km}$ by $\sim 50 \mathrm{~km}$ are resolvable. The test results indicate that our network can resolve coupling up to $20 \mathrm{~km}$ offshore the Nicoya and Osa peninsulas, including the region 


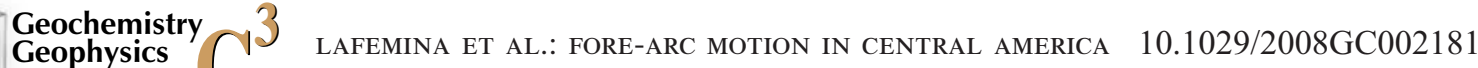
Geosystems
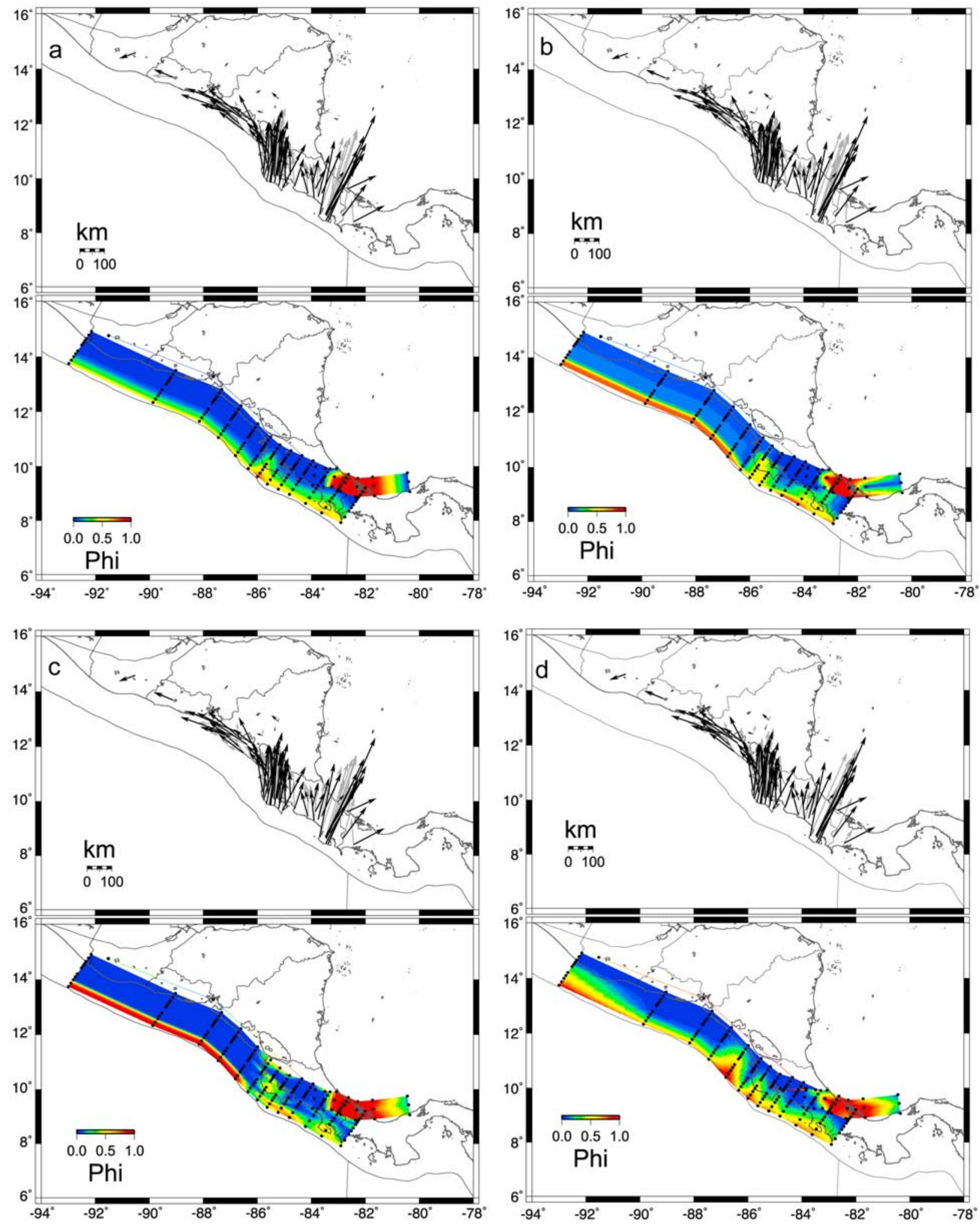

Figure 9 
northwest of Nicoya Peninsula. Our geodetic network, however, has difficulties resolving the pattern of coupling farther offshore in central Costa Rica, where the network does not have high enough spatial density on the coast, and in Nicaragua, where the fore arc is more than $150 \mathrm{~km}$ from the trench (Figure 8b).

[24] Studies of interseismic elastic strain accumulation at subduction zones have demonstrated the inability of geodetic networks to fully resolve the updip limit of seismogenic zone coupling offshore and out to the trench [McCaffrey, 2002; Wang et $a l ., 2003]$. Analyses of slip partitioning models by McCaffrey et al. [2000] and McCaffrey [2002] suggest that if the plate boundary is not fully coupled $(\varphi=1.0)$ out to the trench, anomalously high strain rates are produced in the outer fore arc. To address these issues we tried several modeling approaches (Table 3 ). In models 1 and 2 the distribution of locking on the fault plane is constrained as follows. In model 1, the fault plane is fully coupled at the trench, and the downdip pattern of coupling is based on the effective transition zone model of Wang et al. [2003]. The fault plane is fully coupled from the surface to a depth, $Z_{1}$, then coupling decays exponentially to a depth, $Z_{2}$ (Figure $9 a$ ). Values for $Z_{1}$ and $Z_{2}$ are varied over a limited range. In model 2 (Figure 9b), the fault plane is fully coupled $(\varphi=1)$ at the trench and decreases linearly to $\varphi=0$ at the downdip end of the seismogenic zone. In model 3 (Figure 9c) the distribution of locking is not constrained. For both models 2 and 3, the pattern of coupling is constrained north of Nicoya Peninsula, where nodes are constrained not to vary along strike because of the limited data in this region. Model 4 (Figure 9d) is similar to model 1 , but the fore arc is split into two independent blocks; a smaller fore-arc block and a Panama block, separated by the Central Costa Rica Deformed Belt (Figures 1 and 9d). Table 3 presents the relative plate rates and azimuths for the best fit models.

\subsection{Coupling Along the Central America Seismogenic Zone in Costa Rica}

[25] All four models indicate the following: (1) the highest resolvable coupling is centered along the central Nicoya segment, (2) coupling along the Nicoya and Osa segments of the Central America seismogenic zone is roughly $50 \%$ of the total convergence rate $(\varphi \cong 0.5)$, (3) coupling on the Central America seismogenic zone at the latitude of Osa Peninsula is over a broad $(\sim 100 \mathrm{~km})$ region, (4) coupling offshore Nicaragua must be shallow (i.e., less than $20 \mathrm{~km}$ depth), and (5) there are obvious spatial differences in the coupling pattern between subducting EPR and CNS lithosphere. However, there are important differences in the estimated patterns of coupling and relative plate and block rates (Table 3). Models 1 and 2 have an elongate zone of $\sim 50 \%$ coupling $(\varphi \cong 0.5)$ from the trench to beneath Nicoya Peninsula (Figures 9a and $9 \mathrm{~b}$ ). The results of the effective transition zone models indicate that ranges in depth $Z_{1}$ of $0-20 \mathrm{~km}$ and depth $Z_{2}$ of $25-40 \mathrm{~km}$ fit the data equally well. In the best fit model (model 1), $Z_{1}=20 \mathrm{~km}$ and $\mathrm{Z}_{2}=40 \mathrm{~km}$ (Figure 9a). Model 3 results in a patch of $50 \%$ coupling directly beneath Nicoya Peninsula (Figure 9c). This result differs somewhat from that of Norabuena et al. [2004] in that the patch is located beneath, not offshore Nicoya Peninsula. The results of the two studies may differ because

Figure 9. Best fit results for models 1 to 4 . Observed (black) and modeled (gray) velocities indicate good fits to the velocity field in the outer fore-arc Nicoya Peninsula and area of fore-arc motion to the northwest. However, the models do not reproduce well the velocity field northwest and southeast of Osa Peninsula and Cocos Ridge. All models indicate roughly $50 \%$ coupling offshore Nicoya and Osa peninsulas and a broad region of coupling at Osa Peninsula. Shallow coupling is required offshore Nicaragua in all models. Northwest of Nicoya Peninsula, the pattern of coupling is constrained for models 2 and 3; nodes along strike have the same coupling value. We define coupling in this region because of the inability of our network to resolve the pattern of coupling offshore. (a) Model 1 (effective transition zone) predicts an elongate zone of coupling from the trench to underneath Nicoya Peninsula. Coupling offshore Nicaragua is shallow. (b) Model 2 (linear decrease in coupling) predicts an elongate zone of coupling from the trench to underneath Nicoya Peninsula. Coupling offshore Nicaragua is shallow but stronger $(\varphi>75 \%)$ than model 1 and defined by a linear decrease in coupling downdip. This model predicts a patch of $50 \%$ coupling from the trench to beneath Nicoya Peninsula. (c) Model 3 (unconstrained) with coupling not constrained from the northern end of Nicoya Peninsula to the southeast edge of the model domain. This model predicts a patch of $50 \%$ coupling beneath Nicoya Peninsula. (d) Model 4 is an effective transition zone model, with additional Panama block boundary. This model results in a larger misfit in central Costa Rica and the Nicaraguan fore arc and back arc. The former is caused by increased sinistral shear across the northeast trending Panama block boundary, here defined as the Central Costa Rican Deformed Belt of Marshall et al. [2000] (see Figure 1 for boundary geometry). The latter is the result of a patch of $\sim 50 \%$ coupling northwest of Nicoya Peninsula. 
Table 3. Best Fit Relative Rates and Azimuths for Given Block Pairs for Models 1-4

\begin{tabular}{|c|c|c|c|c|c|c|c|c|c|}
\hline \multirow[b]{2}{*}{ Model } & \multirow[b]{2}{*}{ Block Pairs $^{\mathrm{a}}$} & \multirow[b]{2}{*}{ Longitude } & \multirow[b]{2}{*}{ Latitude } & \multicolumn{2}{|c|}{$\begin{array}{l}\text { Velocity } \\
\left(\mathrm{mm} \mathrm{a}^{-1}\right)\end{array}$} & \multirow[b]{2}{*}{ NE Correlation } & \multirow{2}{*}{$\begin{array}{c}\text { Rate } \\
\left(\mathrm{mm} \mathrm{a}^{-1}\right)\end{array}$} & \multirow{2}{*}{$\begin{array}{l}\text { Azimuth } \\
\text { (deg) }\end{array}$} & \multirow[b]{2}{*}{$\chi_{\mathrm{v}}{ }^{2 \mathrm{~b}}$} \\
\hline & & & & East & North & & & & \\
\hline Model 1 & & & & & & & & & 1.617 \\
\hline \multirow[t]{3}{*}{ Osa } & COCO_CARI & -84 & 8 & $37.8 \pm 0.0$ & $82.2 \pm 0.0$ & 0.0 & $90.5 \pm 0.0$ & $24.7 \pm 0.0$ & \\
\hline & COCO_FORE & -84 & 8 & $40.0 \pm 3.5$ & $75.2 \pm 3.5$ & 0.5653 & $85.1 \pm 4.2$ & $28.0 \pm 2.3$ & \\
\hline & FORE CARI & -83.5 & 9 & $-3.6 \pm 2.3$ & $7.8 \pm 4.0$ & 0.5034 & $8.6 \pm 3.2$ & $-24.6 \pm 19.5$ & \\
\hline \multirow[t]{3}{*}{ Nicoya } & COCO_CARI & -86.5 & 9 & $35.4 \pm 0.0$ & $77.8 \pm 0.0$ & 0.0 & $85.4 \pm 0.0$ & $24.5 \pm 0.0$ & \\
\hline & COCO_FORE & -86.5 & 9 & $39.0 \pm 2.3$ & $74.1 \pm 2.8$ & -0.3919 & $83.8 \pm 2.2$ & $27.8 \pm 1.7$ & \\
\hline & FORE_CARI & -85 & 10.5 & $-5.7 \pm 1.6$ & $5.7 \pm 2.7$ & -0.2354 & $8.0 \pm 2.5$ & $-44.8 \pm 15.5$ & \\
\hline Nicaragua & FORE_CARI & -86.25 & 12 & $-7.8 \pm 3.0$ & $4.0 \pm 2.6$ & 0.1763 & $8.7 \pm 2.8$ & $-62.9 \pm 17.3$ & \\
\hline El Salvador & FORE_CARI & -89 & 13 & $-9.2 \pm 4.3$ & $0.2 \pm 5.3$ & 0.7892 & $9.2 \pm 4.2$ & $-89.0 \pm 19.4$ & \\
\hline Model 2 & & & & & & & & & 2.680 \\
\hline \multirow[t]{3}{*}{ Osa } & COCO_CARI & -84 & 8 & $37.8 \pm 0.0$ & $82.2 \pm 0.0$ & 0.0 & $90.5 \pm 0.0$ & $24.7 \pm 0.0$ & \\
\hline & COCO_FORE & -84 & 8 & $37.4 \pm 19.6$ & $76.6 \pm 19.7$ & 0.9451 & $85.3 \pm 26.0$ & $26.1 \pm 13.3$ & \\
\hline & FORE_CARI & -83.5 & 9 & $-2.2 \pm 13.8$ & $6.9 \pm 22.4$ & 0.9646 & $7.3 \pm 17.3$ & $-17.7 \pm 38.8$ & \\
\hline \multirow[t]{3}{*}{ Nicoya } & COCO_CARI & -86.5 & 9 & $35.4 \pm 0.0$ & $77.8 \pm 0.0$ & 0.0 & $85.4 \pm 0.0$ & $24.5 \pm 0.0$ & \\
\hline & COCO_FORE & -86.5 & 9 & $37.6 \pm 13.7$ & $78.5 \pm 9.8$ & 0.4958 & $87.1 \pm 12.9$ & $25.6 \pm 8.7$ & \\
\hline & FORE_CARI & -85 & 10.5 & $-6.1 \pm 6.4$ & $3.1 \pm 14.7$ & 0.8498 & $6.9 \pm 3.5$ & $-63.4 \pm 27.8$ & \\
\hline Nicaragua & FORE_CARI & -86.25 & 12 & $-10.0 \pm 7.7$ & $-0.1 \pm 10.3$ & 0.2368 & $10.0 \pm 7.8$ & $269.2 \pm 23.7$ & \\
\hline El Salvador & FORE_CARI & -89 & 13 & $-12.6 \pm 12.9$ & $-7.2 \pm 16.1$ & 0.9216 & $14.5 \pm 18.8$ & $240.1 \pm 29.6$ & \\
\hline Model 3 & & & & & & & & & 1.815 \\
\hline \multirow[t]{3}{*}{ Osa } & COCO_CARI & -84 & 8 & $37.8 \pm 0.0$ & $82.2 \pm 0.0$ & 0.0 & $90.5 \pm 0.0$ & $24.7 \pm 0.0$ & \\
\hline & COCO_FORE & -84 & 8 & $37.2 \pm 15.2$ & $74.9 \pm 14.0$ & 0.9501 & $83.7 \pm 19.1$ & $26.4 \pm 10.5$ & \\
\hline & FORE_CARI & -83.5 & 9 & $-1.8 \pm 10.5$ & $8.5 \pm 16.3$ & 0.9437 & $8.7 \pm 14.0$ & $-11.7 \pm 37.2$ & \\
\hline \multirow[t]{3}{*}{ Nicoya } & COCO_CARI & -86.5 & 9 & $35.4 \pm 0.0$ & $77.8 \pm 0.0$ & 0.0 & $85.4 \pm 0.0$ & $24.5 \pm 0.0$ & \\
\hline & COCO_FORE & -86.5 & 9 & $37.2 \pm 10.4$ & $76.4 \pm 4.2$ & 0.3665 & $84.9 \pm 6.9$ & $26.0 \pm 6.5$ & \\
\hline & FORE_CARI & -85 & 10.5 & $-5.4 \pm 3.9$ & $5.0 \pm 9.5$ & 0.6737 & $7.3 \pm 5.0$ & $-47.4 \pm 26.1$ & \\
\hline Nicaragua & FORE_CARI & -86.25 & 12 & $-9.0 \pm 5.4$ & $2.0 \pm 4.7$ & -0.4236 & $9.2 \pm 5.8$ & $-77.5 \pm 22.2$ & \\
\hline El Salvador & FORE_CARI & -89 & 13 & $-11.4 \pm 9.9$ & $-4.5 \pm 11.2$ & 0.9101 & $12.2 \pm 13.1$ & $248.2 \pm 27.4$ & \\
\hline Model 4 & & & & & & & & & 1.821 \\
\hline \multirow[t]{4}{*}{ Osa } & COCO_CARI & -84 & 8 & $37.8 \pm 0.0$ & $82.2 \pm 0.0$ & 0.0 & $90.5 \pm 0.0$ & $24.7 \pm 0.0$ & \\
\hline & COCO_PANA & -84 & 8 & $35.2 \pm 20.1$ & $80.1 \pm 11.1$ & 0.3898 & $87.5 \pm 15.3$ & $23.7 \pm 12.2$ & \\
\hline & PANA_CARI & -83.5 & 9 & $0.9 \pm 7.2$ & $3.0 \pm 14.7$ & 0.7545 & $3.2 \pm 15.7$ & $17.4 \pm 39.2$ & \\
\hline & PANA_FORE & -84 & 9 & $5.8 \pm 8.2$ & $-3.0 \pm 12.3$ & 0.663 & $6.6 \pm 5.5$ & $117.3 \pm 47.9$ & \\
\hline \multirow[t]{3}{*}{ Nicoya } & COCO_CARI & -86.5 & 9 & $35.4 \pm 0.0$ & $77.8 \pm 0.0$ & 0.0 & $85.4 \pm 0.0$ & $24.5 \pm 0.0$ & \\
\hline & COCO_FORE & -86.5 & 9 & $40.3 \pm 4.0$ & $76.3 \pm 3.7$ & -0.2158 & $86.3 \pm 3.4$ & $27.9 \pm 2.6$ & \\
\hline & FORE_CARI & -85 & 10.5 & $-7.2 \pm 2.5$ & $3.7 \pm 4.0$ & 0.0409 & $8.1 \pm 2.8$ & $-62.7 \pm 18.3$ & \\
\hline Nicaragua & FORE_CARI & -86.25 & 12 & $-9.4 \pm 3.8$ & $1.8 \pm 3.6$ & 0.1338 & $9.6 \pm 3.7$ & $-78.9 \pm 16.6$ & \\
\hline El Salvador & FORE_CARI & -89 & 13 & $-10.9 \pm 5.4$ & $-2.3 \pm 6.9$ & 0.7625 & $11.1 \pm 6.4$ & $258.3 \pm 21.6$ & \\
\hline
\end{tabular}

\footnotetext{
${ }^{\mathrm{a}}$ Blocks are Central American fore arc (FORE), Caribbean plate (CARI), Cocos plate (COCO), and Panama (PANA). Rates and azimuths are for first block relative to second block.

${ }^{\mathrm{b}}$ Reduced chi square.
}

here we simultaneously inverted for coupling and block motion, instead of calculating them independently as was done by Norabuena et al. [2004]. In addition, the data sets are substantially different. We use approximately double the number of GPS sites and both horizontal and vertical velocities; Norabuena et al. [2004] used only the horizontal. Note that our EGPS measurements will not necessarily capture all interseismic strain if slow slip events are common here. In this case our coupling estimates should be considered minimum estimates.
[26] Our new estimates of coupling on the plate interface allow us to estimate the amount of elastic strain (or slip deficit) accumulated since the last earthquake on a given segment of the plate boundary. These estimates assume a simple model of steady elastic strain accumulation. Strain sampled by the EGPS data is assumed to be representative of the entire seismic cycle, and earthquakes are assumed to be strictly periodic and have occurred on the same fault segments. With these assumptions, it appears that there is a slip deficit on the Osa segment despite three recent large earthquakes. 
The plate boundary is currently accumulating slip deficit at a rate of $\sim 44 \mathrm{~mm} \mathrm{a}^{-1}$, i.e., $\sim 50 \%$ coupling at a rate of $\sim 87 \mathrm{~mm} \mathrm{a}^{-1}$ (Figure 9 and Table 3, model 1). For the 43 years between the 1940 and 1983 earthquakes, a slip deficit of $\sim 1.9 \mathrm{~m}$ accumulated. Tajima and Kikuchi [1995] estimated only $0.6 \mathrm{~m}$ of slip for the $1983 M_{w} 7.3$ earthquake. This indicates a slip deficit of $\sim 1.3 \mathrm{~m}$ for that earthquake. Approximately $0.9 \mathrm{~m}$ has accumulated since 1983 for a total of $2.2 \mathrm{~m}$. This suggests that the Osa segment is either "ready" for a large magnitude earthquake or that strain is being released aseismically, either as afterslip following $M>7$ earthquakes (prior to our measurements) or during transient slow slip events. An alternate interpretation of these data is presented in the following section.

[27] Similarly, the Nicoya segment last had a large earthquake in 1950. Our new model results (Figure 9 and Table 3) suggest that it is accumulating a slip deficit at a rate up to $\sim 42 \mathrm{~mm} \mathrm{a}^{-1}$ (i.e., $\sim 50 \%$ coupling at a rate of $\sim 84 \mathrm{~mm} \mathrm{a}^{-1}$ ), indicating an accumulation of $\sim 2.2 \mathrm{~m}$ of slip deficit, presumably to be released during the next earthquake. Again, transient slow slip events unrecorded by our EGPS observations [e.g., Protti et al., 2004], may relieve some fraction of the accumulated slip deficit.

\subsection{Coupling Along the Central America Seismogenic Zone Offshore Nicaragua}

[28] The velocity field in the Nicaraguan fore arc indicates significant margin-parallel motion; there is essentially no margin-normal velocity recorded here (Figures 4 and 5). Since plate boundary earthquakes occur offshore Nicaragua, this segment must be coupled, but our models restrict coupling to shallow depths $(<20 \mathrm{~km})$; deeper locking is not allowed by the data. The last major plate boundary earthquake, the $1992 \mathrm{M}_{\mathrm{s}} 7.2$ tsunami earthquake, had a focal mechanism indicating pure thrusting, and a shallow rupture plane, less than $20 \mathrm{~km}$ depth [Ide et al., 1993; Satake, 1994]. This implies strain accumulation prior to this event more than $80 \mathrm{~km}$ offshore, beyond the resolution of our terrestrial network (Figure 8b).

\subsection{Fore-Arc Motion in Central America}

[29] Our new velocity field and modeling results (Figure 9 and Table 3) indicate trench-parallel forearc motion from central Costa Rica to El Salvador and Guatemala and allow for improved estimates of this motion. The velocity field indicates fore-arc motion to the east-northeast into western Panama, but this is not predicted by any of the four models (Figures 9a-9d). The best fit model (model 1) indicates northwest fore-arc motion at rates of $\sim 8 \mathrm{~mm} \mathrm{a}^{-1}$ in northern Costa Rica to $\sim 9 \mathrm{~mm} \mathrm{a}^{-1}$ in El Salvador (Table 3). In addition, the azimuth of fore-arc motion rotates counterclockwise, following the plate boundary. This rotation is defined by the estimated Euler vector for the Central American fore-arc block relative to the Caribbean plate (E 269.85, N 3.90, $0.469^{\circ} / \mathrm{Ma}$ ).

[30] While our block model assumes discrete, piecewise planar boundaries (Figure 7), in fact the Caribbean-fore arc boundary is more likely a diffuse, deforming zone dominated by dextral shear [McCaffrey, 1996; La Femina et al., 2002], but accommodated by combined block rotation and extension, not reflected in our model. We tested the effects of splitting the fore arc into two discrete tectonic blocks separated by the Central Costa Rica Deformed Belt as described by Marshall et al. [2000]. Figure 9d and Table 3 give the results of model 4. This model suggests higher rates of forearc motion, but did not significantly improve the misfit.

[31] Fore-arc motion in Central America was first postulated by Molnar and Sykes [1969] on the basis of focal mechanism solutions of intraplate (arc and fore arc) earthquakes. Fore-arc motion has more recently been quantified through analysis of the deflection of interplate earthquake slip vectors [McCaffrey, 1992, 1996] and geodetic data. DeMets [2001] estimated a fore-arc rate of $14 \pm 2 \mathrm{~mm} \mathrm{a}^{-1}$ between Nicaragua and Guatemala based on a new estimate of Cocos-Caribbean plate motion. McCaffrey [2002] utilized GPS velocities presented by Lundgren et al. [1999] and earthquake slip vectors to solve simultaneously for plate coupling and motion of a fore-arc block, estimating a fore-arc translation rate of $5.8 \pm 5.5 \mathrm{~mm} \mathrm{a}^{-1}$ at the latitude of Nicoya peninsula. Norabuena et al. [2004] used an improved GPS velocity field and estimated fore-arc motion of $8 \pm 3 \mathrm{~mm} \mathrm{a}^{-1}$ in the Nicoya region. Using GPS sites in the Nicaraguan fore arc, Turner et al. [2007] estimated a rate of arc-parallel motion up to $15 \mathrm{~mm} \mathrm{a}^{-1}$ in northern Nicaragua assuming a dextral strike-slip boundary oriented N50W. Our best fit model (model 1, Table 3), which includes all the geodetic observations from the region and earthquake slip vectors, indicates $7.7 \pm 2.5 \mathrm{~mm} \mathrm{a}^{-1}$ at the latitude of Nicoya Peninsula, $8.4 \pm 3.0 \mathrm{~mm} \mathrm{a}^{-1}$ in central Nicaragua and $8.8 \pm 4.3 \mathrm{~mm} \mathrm{a}^{-1}$ in El Salvador suggesting no significant change in rate of fore-arc motion with 


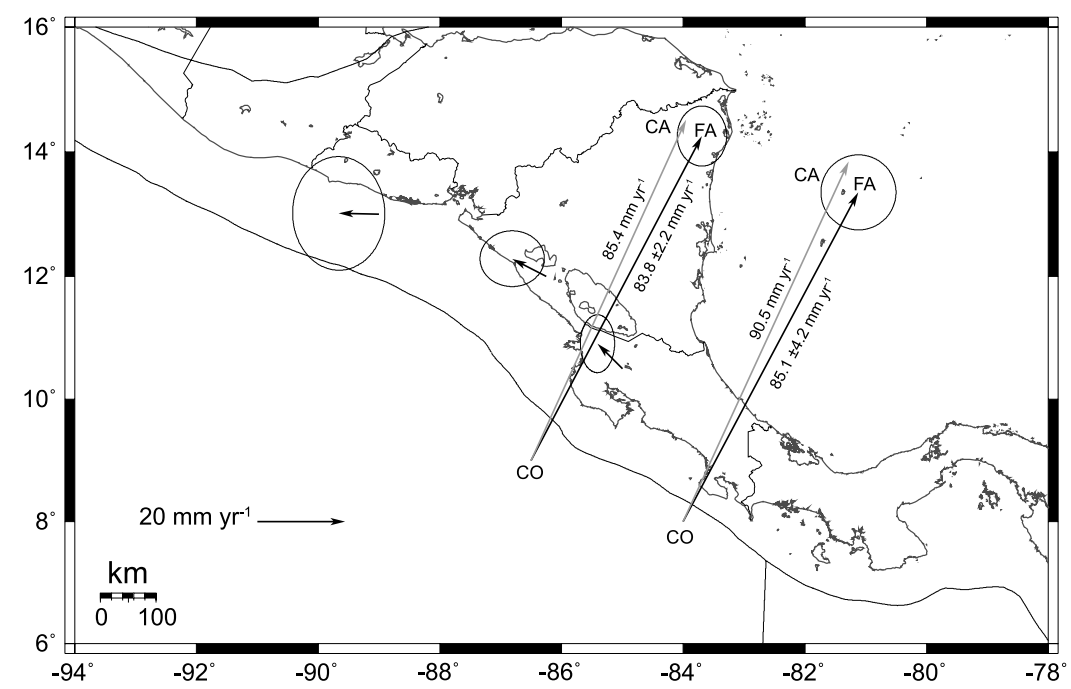

Figure 10. Relative plate and block motion rates between the Central American fore-arc block and the Caribbean plate (black vectors with 1-sigma uncertainty ellipses) (model 1, Table 3). Velocity triangle diagrams showing relative Cocos-fore arc motion (black vectors with 1-sigma uncertainties) and the Cocos-Caribbean relative plate vector of DeMets [2001] (gray vector) at the latitudes of Osa and Nicoya peninsulas.

latitude (Figure 10). Model 4 indicates a change in rate from $8.1 \pm 2.8 \mathrm{~mm} \mathrm{a}^{-1}$ at the latitude of Nicoya Peninsula, to $9.6 \pm 3.7 \mathrm{~mm} \mathrm{a}^{-1}$ in central Nicaragua and $11.1 \pm 6.4 \mathrm{~mm} \mathrm{a}^{-1}$ in El Salvador.

[32] The motion of rigid fore-arc blocks results in the formation of both trailing and leading edges. The trailing edge may be expressed as a zone of lithospheric extension. Lewis et al. [2008] indicate that the trailing edge of this system is located in central Costa Rica on the basis of an analysis of background seismicity. The leading edge maybe expressed as a zone of convergence. For the Central American fore arc, the leading edge is an unstable plate boundary where four plates come together: the Caribbean, North American and Cocos plates and the Central American fore-arc block. The North American-Caribbean boundary becomes diffuse at this latitude and shortening occurs across the boundary. The shortening direction, however, is roughly normal to the motion of the fore arc [Guzman-Speziale and Meneses-Rocha, 2000]. As we model and describe below, fore-arc translation in Central America may be the result of Cocos Ridge collision. This process initiated after $5 \mathrm{Ma}$ and could be as young as $0.5 \mathrm{Ma}$ [MacMillan et al., 2004, and references therein]. Hence, the fore-arc system is still in the incipient stage of development and there has been a maximum of $\sim 45 \mathrm{~km}$ or minimum of $\sim 4.5 \mathrm{~km}$ of block translation and convergence. Furthermore, there is no well-defined margin-parallel strike slip fault as might be expected in a more tectonically mature system.

\subsection{Deformation in Southern Costa Rica}

[33] Plate motion models estimate the CocosCaribbean relative convergence rate at the latitude of Osa Peninsula is $90.5 \mathrm{~mm} \mathrm{a}^{-1}$ with an azimuth of $\sim 26^{\circ}$ [DeMets, 2001] (Figure 10). Our threedimensional block model predicts Cocos-fore arc relative motion here to be $\sim 84-87 \mathrm{~mm} \mathrm{a}^{-1}$ at an azimuth of $24^{\circ}$ to $28^{\circ}$ (Table 3 ). The best fit estimate is $85.1 \pm 4.2 \mathrm{~mm} \mathrm{a}^{-1}$ at an azimuth of $28 \pm 2^{\circ}$ (Table 3 , model 1). Our best estimate of Caribbean-fore arc motion, $8.6 \pm 3.2 \mathrm{~mm} \mathrm{a}^{-1}$ at an azimuth of $-24 \pm 20^{\circ}$ (Table 3 , model 1), is similar to earlier estimates [Plafker and Ward, 1992; Dixon, 1993; Suarez et al., 1995]. Recent geologic studies of shortening across the Fila Costena fold and thrust belt estimate $10-40 \mathrm{~mm} \mathrm{a}^{-1}$ of Quaternary shortening [Fisher et al., 2004; Sitchler et al., 2007]. This suggests that permanent shortening constitutes a significant fraction of Cocos-fore arc relative motion here.

[34] The elastic block models are not able to reproduce the counterclockwise and clockwise rotation of velocity vectors away from Cocos Ridge observed in central and southeastern Costa Rica and western Panama, nor predict fore-arc motion in areas where convergence is orthogonal to the trench. The surface velocity field has a distinct radial pattern away from the point where Cocos Ridge 
contacts the margin (Figure 4), indicating that the upper crust is moving away from this region. Thickened CNS-2 and Cocos Ridge crust may act as a rigid or semirigid indenter. Fore-arc motion reflects tectonic escape from the rigid indenter. We test this scenario in the next section.

\subsection{Geologic Deformation Model}

[35] It has long been recognized that regions of oblique plate convergence may be associated with trench-parallel motion of a coastal block (fore arc), between the trench and volcanic arc [Fitch, 1972; Jarrard, 1986]. It is usually assumed that oblique convergence drives this fore-arc motion directly, via frictional coupling across the seismogenic portion of the plate interface. In this case, we might expect that the presence or absence of trench-parallel fore-arc motion, as well as the speed of the fore arc, is related to factors such as rate and obliquity of convergence, the degree of plate coupling, and the existence of a weak zone to accommodate fore-arc motion, e.g., thermally weakened crust associated with the volcanic arc [Beck, 1983; Jarrard, 1986; Beck, 1991; McCaffrey, 1992]. However, as discussed in the previous section, seismic coupling is low along most of the Nicaraguan segment of the Middle America margin, and fore-arc translation occurs even in southern Costa Rica where convergence is orthogonal. This suggests that oblique convergence may not be the only driving mechanism for fore-arc motion here.

[36] Subduction of seafloor bathymetric highs can have a profound effect on the subduction process and on upper plate deformation. Young and/or thickened oceanic lithosphere, including aseismic ridges like the Cocos Ridge or the D'Entrecasteaux Ridge offshore the New Hebrides, resist subduction due to buoyancy forces [Vogt et al., 1976; McCann and Habermann, 1989; Cloos, 1993] resulting in the evolution of a convergent margin from subduction to collision and permanent strain in upper plate lithosphere [e.g., Gardner et al., 2001; Fisher et $a l ., 2004]$. On the basis of studies of upper plate deformation, it has been suggested that the Cocos Ridge acts as a rigid indenter, colliding with the Central American isthmus [Corrigan et al., 1990; Gardner et al., 1992; Kolarsky et al., 1995]. Whether the Cocos Ridge is better considered to subduct or collide is not clear; while earthquakes clearly delineate a dipping slab in the north, at the latitude of the Cocos Ridge the slab can only be clearly followed to a depth of $60 \mathrm{~km}$ [Protti et al., 1994]. The collision model presented here can therefore be considered as one end-member, designed to test whether tectonic escape from the indenter is a viable driving force for fore-arc motion.

[37] We use a finite element (FE) model (G-TECTON [Govers and Meijer, 2001]) to investigate long-term regional horizontal strain, modeling the collision of young, hot spot-thickened oceanic crust (CNS-2Cocos Ridge crust) with southern Central America. This model differs substantially from the more conventional subduction strain accumulation models described above. Our FE model domain (Figure 11) consists of spherical shell elements with laterally varying rheology. The Caribbean plate, including the Nicaraguan and Honduran highlands, is treated as elastic lithosphere (Young's modulus $5 \times 10^{4} \mathrm{MPa}$, Poisson ratio 0.3 ), consistent with geodetic and geologic observations for a rigid Caribbean plate [Sella et al., 2002; Lopez et al., 2006]. The volcanic arc and fore arc are treated as viscoelastic lithosphere (viscosity $10^{19} \mathrm{~Pa}$ s, Young's modulus $5 \times$ $10^{4} \mathrm{MPa}$, Poisson ratio 0.3; results are shown after 12 Maxwell times). The model domain is fixed along the northern boundary, which crosses the stable Caribbean plate, and free along the eastern and western boundaries, in agreement with interaction of the Caribbean plate with the North and South American plates [Dixon and Mao, 1997] (Figure 11). The model includes two low-friction strike slip faults through the volcanic arc (in Costa Rica and Nicaragua-El Salvador, respectively) that allow strike-slip motion if required by model stresses. We use a velocity boundary condition equal to the Cocos-Caribbean convergence rate $\left(90.5 \mathrm{~mm} \mathrm{a}^{-1}\right)$ to drive deformation along the MAT in the region of CNS-2-Cocos Ridge lithosphere (Figures $3 \mathrm{a}$ and 11).

[38] Figure 11 compares modeled and observed horizontal velocities. The model clearly captures the main features of the velocity field. In particular, it matches the observed high displacement rates inboard of Cocos Ridge, the rotation of vectors away from the ridge, and northwestward translation of the fore arc. We interpret this agreement to indicate that Cocos Ridge collision and subduction of thickened CNS-2 crust is a viable mechanism for driving tectonic escape and translation of the Central American fore arc, as well as permanent fore-arc shortening in the Fila Costena. Of course, this does not preclude other driving mechanisms from contributing as well. Our model represents only one end-member process (Cocos Ridge collision) and does not incorporate all of the relevant processes that may drive or resist fore-arc motion. 


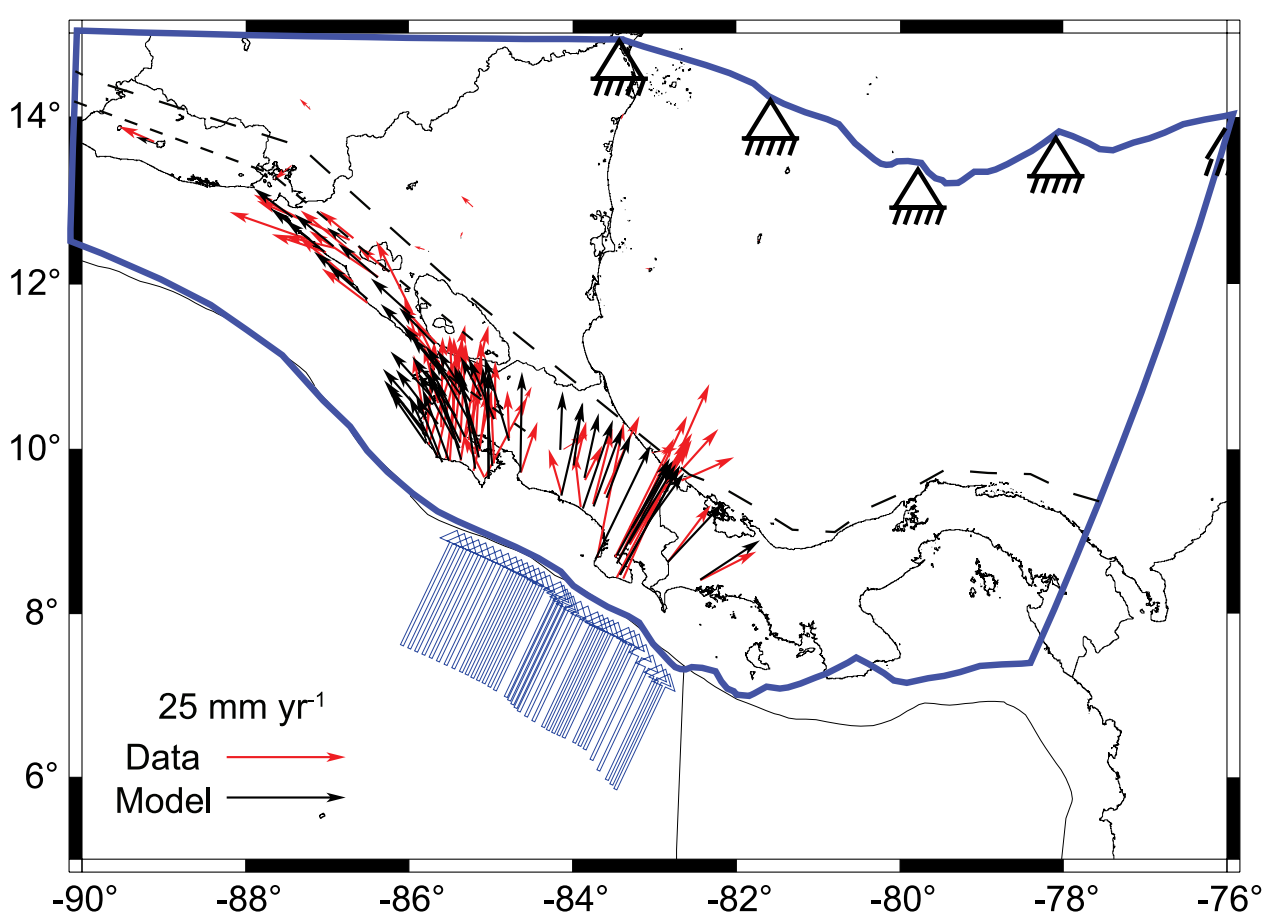

Figure 11. Finite element model with observed (red) versus calculated (black) horizontal velocities. Model domain is indicated by blue line, and open triangles with feet indicate fixed northern boundary. Open blue vectors indicate zone of forcing along the MAT. Faults near the volcanic arc (black dotted lines) accommodate fore-arc sliver transport. Dashed black line in back arc represents rheological boundary (see text for further discussion).

These include subduction-related coupling and resultant strain accumulation, potential kinematic and dynamic effects of the semi-independent Panama block, and the effects of trench-parallel flow in the upper mantle [e.g., Hoernle et al., 2008], all of which also likely affect the surface velocity field and the magnitude and distribution of forces affecting the fore arc.

\section{Discussion}

[39] The advent of high-precision GPS measurements for quantifying surface displacements has enabled an increasingly detailed picture of lithospheric deformation. In particular, it is becoming possible to address a long-standing question in continental tectonics, namely whether crustal deformation is best modeled as continuum versus block deformation [Thatcher, 1995]. The latter view essentially adopts the plate tectonics paradigm, where the crust is considered rigid, except where broken by faults. Faults behave as narrow weak zones over long time scales, focusing deformation and accommodating relative motion between blocks. On short time scales, the faults may be locked and elastic strain accumulation modifies the long-term velocity of sites within several lithospheric thicknesses of the fault. In our view, the block model is an excellent firstorder description of most continental deformation zones, which evolve to exploit zones of weaknesses that, because of the strain-weakening rheology of crustal materials, eventually become well-defined, block-bounding faults. Our numerical model is a continuum model, but also fits the observed deformation field quite well. This may reflect the fact that Cocos Ridge collision is a recent phenomenon, initiating within the last 5 million years, and possibly as young as $0.5 \mathrm{Ma}$ [e.g., MacMillan et al., 2004, and references therein], and the region has not yet had time to evolve into a set of blocks with well-defined fault boundaries. In particular, the boundary between the fore arc and Caribbean plate from central Costa Rica to El Salvador is a diffuse zone of conjugate faulting rather than a well-defined margin-parallel strike slip fault like the Sumatra fault. Furthermore, the western boundary of the Panama block (i.e., the Central Costa Rican Deformed Belt) lacks a clearly defined, throughgoing fault [Marshall et al., 2000]. A well-defined fore arc-Caribbean boundary will presumably develop in the future, perhaps leading to 
an increased rate of fore-arc motion as fault resistance decreases.

\section{Conclusions}

[40] A new regional surface velocity field for Central America reflects interaction between the Cocos and Caribbean plates. Most site velocities reflect two approximately orthogonal components. Northeast motion, in the direction of plate convergence, occurs at rates up to $44 \mathrm{~mm} \mathrm{a}^{-1}$ relative to the interior of the Caribbean plate, and is best developed in the Osa and Nicoya peninsulas, Costa Rica. Northwest motion, parallel to the margin, is best exhibited by sites in northern Costa Rica and Nicaragua, and averages $8 \mathrm{~mm} \mathrm{a}^{-1}$.

[41] We interpret the northeast component to largely reflect the short-term, elastic response to locking on the boundary between the subducting Cocos plate and overriding Central American fore-arc block. We interpret the northwest component to reflect long-term translation of the fore-arc block. This translation is driven by either or both of two processes: (1) oblique convergence of the Cocos plate and slip partitioning and/or (2) collision of the Cocos Ridge with the Central America fore arc, whereby the ridge acts as a rigid or semirigid indenter, and fore-arc motion represents a form of tectonic escape.

\section{Acknowledgments}

[42] This work was supported by NSF EAR and Margins grants and several NASA grants to T. H. D. and a NASA Space Grant Fellowship to P. L. Additional support for G. S. M. was obtained from NSF EAR grants 0538135 and 0703790 . H. L. T. was supported by a Walton Distinguished Doctoral Fellowship at University of Arkansas. We thank Rob McCaffrey for making DEFNODE available and for valuable discussions and comments, UNAVCO for assistance with field operations, and Tom Gardner and Kristin Morrell for help with Figure 3. Computational resources for finite element modeling were provided by the Netherlands Research Center for Integrated Solid Earth Science. The Generic Mapping Tools (GMT) software was used in making Figures 1, 2, and 4-11.

\section{References}

Adamek, S., F. Tajima, and D. Wiens (1987), Seismic rupture associated with subduction of the Cocos ridge, Tectonics, 6 , 757-774, doi:10.1029/TC006i006p00757.

Allmendinger, R. W., R. J. Smalley, M. Bevis, H. Caprio, and B. Brooks (2005), Bending the Bolivian orocline in real time, Geology, 33, 905-908, doi:10.1130/G21779.1.

Barckhausen, U., C. R. Ranero, R. von Huene, S. C. Cande, and H. A. Roeser (2001), Revised tectonic boundaries in the Cocos plate off Costa Rica: Implications for segmentation of the convergent margin and for plate tectonic models, J. Geophys. Res., 106, 19,207-19,220, doi:10.1029/2001JB000238.

Beck, M. E. (1983), On the mechanism of tectonic transport in zones of oblique subduction, Tectonophysics, 93, 1-11, doi:10.1016/0040-1951(83)90230-5.

Beck, M. E. (1991), Coastwise transport reconsidered: Lateral displacements in oblique subduction zones, and tectonic consequences, Phys. Earth Planet. Inter., 68, 1-8, doi:10.1016/ 0031-9201(91)90002-Y.

Bilek, S. L., S. Y. Schwartz, and H. R. DeShon (2003), Control of seafloor roughness on earthquake rupture behavior, Geology, 31, 455-458, doi:10.1130/0091-7613(2003)031<0455: COSROE $>2.0 . \mathrm{CO} ; 2$.

Bird, P. (2003), An updated digital model of plate boundaries, Geochem. Geophys. Geosyst., 4(3), 1027, doi:10.1029/ 2001GC000252.

Carr, M. J. (1976), Underthrusting and Quaternary faulting in northern Central America, Geol. Soc. Am. Bull., 87, 825828, doi:10.1130/0016-7606(1976)87<825:UAQFIN $>2.0$. $\mathrm{CO} ; 2$.

Cloos, M. (1993), Lithospheric buoyancy and collisional orogenesis: Subduction of oceanic plateaus, continental margins, island arcs, spreading ridges and seamounts, Geol. Soc. Am. Bull., 105, 715-737, doi:10.1130/00167606(1993)105<0715:LBACOS>2.3.CO;2.

Corrigan, J., P. Mann, and J. C. J. Ingle (1990), Forearc response to subduction of the Cocos Ridge, Panama-Costa Rica, Geol. Soc. Am. Bull., 102, 628-652, doi:10.1130/ 0016-7606(1990)102<0628:FRTSOT>2.3.CO;2.

Corti, G., E. Carminati, F. Mazzarini, and M. O. Garcia (2005), Active strike-slip faulting in El Salvador, Central America, Geology, 33, 989-992, doi:10.1130/G21992.1.

Davis, E. E., and H. W. Villinger (2006), Transient formation fluid pressures and temperatures in the Costa Rica forearc prism and subducting oceanic basement: CORK monitoring at ODP Sites 1253 and 1255, Earth Planet. Sci. Lett., 245, 232-244, doi:10.1016/j.epsl.2006.02.042.

DeMets, C. (2001), A new estimate for present-day CocosCaribbean plate motion: Implications for slip along the Central American volcanic arc, Geophys. Res. Lett., 28, 4043-4046, doi:10.1029/2001GL013518.

DeShon, H. R., S. Y. Schwartz, S. L. Bilek, L. M. Dorman, V. Gonzalez, J. M. Protti, E. R. Flueh, and T. H. Dixon (2003), Seismogenic zone structure of the southern Middle America Trench, Costa Rica, J. Geophys. Res., 108(B10), 2491, doi:10.1029/2002JB002294.

DeShon, H. R., S. Y. Schwartz, A. V. Newman, V. Gonzalez, J. M. Protti, L. M. Dorman, T. Dixon, D. E. Sampson, and E. R. Flueh (2006), Seismogenic zone structure beneath the Nicoya Peninsula, Costa Rica, from three-dimensional local earthquake P- and S-wave tomography, Geophys. J. Int., 164, 109-124, doi:10.1111/j.1365-246X.2005.02809.x.

Dixon, T. H. (1993), GPS measurements of relative motion of the Cocos and Caribbean plates and strain accumulation across the Middle America trench, Geophys. Res. Lett., 20, 2167-2170, doi:10.1029/93GL02415.

Dixon, T. H., and A. Mao (1997), A GPS estimate of relative motion between North and South America, Geophys. Res. Lett., 24, 535-538, doi:10.1029/97GL00284.

Fisher, D. M., T. W. Gardner, J. S. Marshall, P. B. Sak, and M. Protti (1998), Effect of subducting sea-floor roughness on fore-arc kinematics, Pacific Coast, Costa Rica, Geology, 26, 467-470, doi:10.1130/0091-7613(1998)026<0467: EOSSFR $>2.3 . \mathrm{CO} ; 2$.

Fisher, D. M., T. W. Gardner, and P. Sak (2004), Active thrusting in the inner fore arc of an erosive convergent margin, 
Pacific coast, Costa Rica, Tectonics, 23, TC2007, doi:10.1029/2002TC001464.

Fitch, T. J. (1972), Plate convergence, transcurrent faults, and internal deformation adjacent to southeast Asia and western Pacific, J. Geophys. Res., 77, 4432-4460, doi:10.1029/ JB077i023p04432.

Gardner, T. W., D. Verdonck, N. M. Pinter, R. Slingerland, K. P. Furlong, T. F. Bullard, and S. G. Wells (1992), Quaternary uplift astride the aseismic Cocos Ridge, Pacific coast, Costa Rica, Geol. Soc. Am. Bull., 104, 219-232, doi:10.1130/ 0016-7606(1992)104<0219:QUATAC >2.3.CO;2.

Gardner, T., et al. (2001), Holocene forearc block rotation in response to seamount subduction, southeastern Peninsula de Nicoya, Costa Rica, Geology, 29(2), 151-154, doi:10.1130/ 0091-7613(2001)029<0151:HFBRIR>2.0.CO;2.

Goes, S. D. B., A. A. Velasco, S. Y. Schwartz, and T. Lay (1993), The April 22, 1991, Valle de Estrella, Costa Rica $(\mathrm{Mw}=7.7)$ earthquake and its tectonic implications: A broadband seismic study, J. Geophys. Res., 98, 8127-8142, doi:10.1029/ 93JB00019.

Govers, R., and P. T. Meijer (2001), On the dynamics of the Juan de Fuca plate, Earth Planet. Sci. Lett., 189, 115-131, doi:10.1016/S0012-821X(01)00360-0.

Guzman-Speziale, M., and J. J. Meneses-Rocha (2000), The North American-Caribbean plate boundary west of the Motogau-Polochic fault system: A fault jog in southeastern Mexico, J. South Am. Earth Sci., 13, 459-468, doi:10.1016/ S0895-9811(00)00036-5.

Hardy, N. C. (1991), Tectonic evolution of the easternmost Panama Basin: Some new data and inferences, J. South Am. Earth Sci., 4, 261-269, doi:10.1016/0895-9811(91)90035-J.

Hoernle, K., et al. (2008), Arc-parallel flow in the mantle wedge beneath Costa Rica and Nicaragua, Nature, 451, 1094-1097, doi:10.1038/nature06550.

Husen, S., E. Kissling, and R. Quintero (2002), Tomographic evidence for a subducted seamount beneath the Gulf of Nicoya, Costa Rica: The cause of the $1990 \mathrm{Mw}=7.0 \mathrm{Gulf}$ of Nicoya earthquake, Geophys. Res. Lett., 29(8), 1238, doi:10.1029/2001GL014045.

Ide, S., F. Imamura, Y. Yoshida, and K. Abe (1993), Source characteristics of the Nicaraguan tsunami earthquake of September 2, 1992, Geophys. Res. Lett., 20, 863-866, doi:10.1029/93GL00683.

Jarrard, R. D. (1986), Terrane motion by strike-slip faulting of forearc slivers, Geology, 14, 780-783, doi:10.1130/00917613(1986)14<780:TMBSFO >2.0.CO;2.

Kolarsky, R. A., P. Mann, and W. Montero (1995), Island arc response to shallow subduction of the Cocos Ridge, Costa Rica, Spec. Pap. Geol. Soc. Am., 295, 235-262.

La Femina, P. C., T. H. Dixon, and W. Strauch (2002), Bookshelf faulting in Nicaragua, Geology, 30, 751-754, doi:10.1130/0091-7613(2002)030<0751:BFIN>2.0.CO;2.

Larson, K. M., A. R. Lowry, V. Kostoglodov, W. Hutton, O. Sanchez, K. Hudnut, and G. Suarez (2004), Crustal deformation measurements in Guerrero, Mexico, J. Geophys. Res., 109, B04409, doi:10.1029/2003JB002843.

Lewis, J. C., A. C. Boozer, A. Lopez, and W. Montero (2008), Collision versus sliver transport in the hanging wall at the Middle America subduction zone: Constraints from background seismicity in central Costa Rica, Geochem. Geophys. Geosyst., 9, Q07S06, doi:10.1029/2007GC001711.

Lonsdale, P. (2005), Creation of the Cocos and Nazca plates by fission of the Farallon plate, Tectonophysics, 404, 237-264, doi:10.1016/j.tecto.2005.05.011.

Lonsdale, P., and K. D. Klitgord (1978), Structure and tectonic history of the eastern Panama Basin, Geol. Soc. Am. Bull.,
89, 981-999, doi:10.1130/0016-7606(1978)89<981: SATHOT $>2.0 . \mathrm{CO} ; 2$.

Lopez, A. M., S. Stein, T. Dixon, G. Sella, E. Calais, P. Jansma, J. Weber, and P. LaFemina (2006), Is there a northern Lesser Antilles forearc block?, Geophys. Res. Lett., 33, L07313, doi:10.1029/2005GL025293.

Lowrie, A., T. Aitken, P. Grim, and L. McRaney (1979), Fossil spreading center and faults within the Panama Fracture Zone, Mar. Geophys. Res., 4, 153-166, doi:10.1007/BF00286402.

Lundgren, P. R., S. K. Wolf, M. Protti, and K. J. Hurst (1993), GPS measurements of crustal deformation associated with the 22 April 1991, Valle de la Estrella, Costa Rica earthquake, Geophys. Res. Lett., 20, 407-410, doi:10.1029/ 93GL00294.

Lundgren, P., M. Protti, A. Donnellan, M. Heflin, E. Hernandez, and D. Jefferson (1999), Seismic cycle and plate margin deformation in Costa Rica: GPS observations from 1994 to 1997, J. Geophys. Res., 104(B12), 28,915-28,926, doi:10. 1029/1999JB900283.

MacMillan, I., P. B. Gans, and G. Alvarado (2004), Middle Miocene to present plate tectonic history of the southern Central American Volcanic Arc, Tectonophysics, 392, 325 348, doi:10.1016/j.tecto.2004.04.014.

Mann, P., and J. Corrigan (1990), Model for Neogene deformation in Panama, Geology, 18, 558-562, doi:10.1130/ 0091-7613(1990)018<0558:MFLNDI >2.3.CO;2.

Marshall, J. S., D. M. Fisher, and T. W. Gardner (2000), Central Costa Rica deformed belt: Kinematics of diffuse faulting across the western Panama Block, Tectonics, 19, 468-497, doi:10.1029/1999TC001136.

McCaffrey, R. (1992), Oblique plate convergence, slip vectors, and forearc deformation, J. Geophys. Res., 97, 8905-8915, doi:10.1029/92JB00483.

McCaffrey, R. (1996), Estimates of modern arc-parallel strain rates in fore arcs, Geology, 24, 27-30, doi:10.1130/00917613(1996)024<0027:EOMAPS > 2.3.CO; 2 .

McCaffrey, R. (2002), Crustal block rotations and plate coupling, in Plate Boundary Zones, Geodyn. Ser, vol. 30, edited by S. Stein and J. Freymueller, pp. 101-122, AGU, Washington, D. C.

McCaffrey, R., P. C. Zwick, Y. Bock, L. Prawirodirdjo, J. F. Genrich, C. W. Stevens, S. S. O. Puntodewo, and C. Subarya (2000), Strain partitioning during oblique plate convergence in northern Sumatra: Geodetic and seismologic constraints and numerical modeling, J. Geophys. Res., 105(B12), 28,363-28,376, doi:10.1029/1999JB900362.

McCann, W. R., and R. E. Habermann (1989), Morphologic and geologic effects of the subduction of bathymetric highs, Pure Appl. Geophys., 129, 41-69, doi:10.1007/BF00874624. McIntosh, K. D., E. A. Silver, I. Ahmed, A. Berhorst, C. R. Ranero, R. K. Kelly, and E. R. Flueh (2007), The Nicaragua Convergent Margin: Seismic reflection imaging of the source of a tsunami earthquake, in The Seismogenic Zone of Subduction Thrust Faults, edited by T. Dixon and J. C. Moore, pp. 257-287, Columbia Univ. Press, New York.

Meschede, M., U. Barckhausen, and H. U. Worm (1998), Extinct spreading on the Cocos Ridge, Terra Nova, 10, $211-$ 216, doi:10.1046/j.1365-3121.1998.00195.x.

Molnar, P., and L. R. Sykes (1969), Tectonics of the Caribbean and Middle America regions from focal mechanisms and seismicity, Geol. Soc. Am. Bull., 80, 1639-1684, doi:10. 1130/0016-7606(1969)80[1639:TOTCAM]2.0.CO;2.

Newman, A. V., J. M. Protti, L. M. Dorman, S. Y. Schwartz, V. Gonzalez, and H. R. DeShon (2002), Along-strike variability in the seismogenic zone below Nicoya Peninsula, Costa 
Rica, Geophys. Res. Lett., 29(20), 1977, doi:10.1029/ 2002GL015409.

Norabuena, E., et al. (2004), Geodetic and seismic constraints on some seismogenic zone processes in Costa Rica, J. Geophys. Res., 109, B11403, doi:10.1029/2003JB002931.

Plafker, G., and S. N. Ward (1992), Thrust faulting and tectonic uplift along the April 22, 1991 Costa Rica earthquake, Tectonics, 11, 709-718, doi:10.1029/92TC00609.

Protti, M., F. Guendel, and K. McNally (1994), The geometry of the Wadati-Benioff zone under southern Central America and its tectonic significance: Results from a high-resolution local seismographic network, Phys. Earth Planet. Inter., 84, 271-287, doi:10.1016/0031-9201(94)90046-9.

Protti, M., F. Guendel, and K. McNally (1995), Correlation between the age of the subducting Cocos Plate and the geometry of the Wadati-Benioff zone under Nicaragua and Costa Rica, in Geologic and Tectonic Development of the Caribbean Plate Boundary in Southern Central America, edited by P. Mann, Spec. Pap. Geol. Soc. Am., 295, 327-340.

Protti, M., et al. (2004), A creep event on the shallow interface of the Nicoya Peninsula, Costa Rica seismogenic zone, Eos Trans. $A G U, 85(47)$, Fall Meet. Suppl., Abstract S41D-07.

Ranero, C. R., and R. von Huene (2000), Subduction erosion along the Middle America convergent margin, Nature, 404, 748-752, doi: $10.1038 / 35008046$.

Sak, P. B., D. M. Fisher, and T. W. Gardner (2004), Effects of subducting seafloor roughness on upper plate vertical tectonism: Osa Peninsula, Costa Rica, Tectonics, 23, TC1017, doi:10.1029/2002TC001474.

Sallarès, V., J. J. Danobeitia, and E. R. Flueh (2000), Seismic tomography with local earthquakes in Costa Rica, Tectonophysics, 329, 61-78, doi:10.1016/S0040-1951(00)00188-8.

Satake, K. (1994), Mechanism of the 1992 Nicaragua tsunami earthquake, Geophys. Res. Lett., 21, 2519-2522, doi:10.1029/94GL02338.

Savage, J. C. (1983), A dislocation Model of strain accumulation and release at a subduction zone, J. Geophys. Res., 88, 4984-4996, doi:10.1029/JB088iB06p04984.

Sella, G., T. H. Dixon, and A. Mao (2002), REVEL: A model for Recent plate velocities from space geodesy, J. Geophys. Res., 107(B4), 2081, doi:10.1029/2000JB000033.

Silver, E. A., D. L. Reed, J. E. Tagudin, and D. J. Heil (1990), Implications of the north and south Panama thrust belts for the origin of the Panama orocline, Tectonics, 9, 261-281, doi:10.1029/TC009i002p00261.

Sitchler, J. C., D. M. Fisher, T. W. Gardner, and J. M. Protti (2007), Constraints on inner forearc deformation from balanced cross sections, Fila Costeña thrust belt, Costa Rica, Tectonics, 26, TC6012, doi:10.1029/2006TC001949.

Spinelli, G. A., and D. M. Saffer (2004), Along-strike variations in underthrust sediment dewatering on the Nicoya margin, Costa Rica related to the updip limit of seismicity, Geophys. Res. Lett., 31, L04613, doi:10.1029/2003GL018863.
Stavenhagen, A. U., et al. (1996), Crustal structure in southern Costa Rica from seismic wide-angle experiments, Eos Trans. $A G U$, 77(46), Fall Meet. Suppl., F645.

Suarez, G., M. Pardo, J. Dominguez, L. Ponce, W. Montero, I. Boschini, and W. Rojas (1995), The Limon, Costa Rica earthquake of April 22, 1991: Back arc thrusting and collisional tectonics in a subduction environment, Tectonics, 14, 518-530, doi:10.1029/94TC02546.

Tajima, F., and M. Kikuchi (1995), Tectonic implications of the seismic ruptures associated with the 1983 and 1991 Costa Rica earthquakes, in Geologic and Tectonic Development of the Caribbean Plate Boundary in Southern Central America, edited by P. Mann, Spec. Pap. Geol. Soc. Am., 295, 327-340.

Thatcher, W. (1995), Microplate versus continuum description of active tectonic deformation, J. Geophys. Res., 100, 3885 3894, doi:10.1029/94JB03064.

Trenkamp, R., J. N. Kellogg, J. T. Freymueller, and H. Mora (2002), Wide plate margin deformation, southern Central America and northwestern South America, CASA GPS observations, J. South Am. Earth Sci., 15, 157-171, doi:10. 1016/S0895-9811(02)00018-4

Turner, H. L. I., P. LaFemina, A. Saballos, G. S. Mattioli, P. E. Jansma, and T. Dixon (2007), Kinematics of the Nicaraguan forearc from GPS geodesy, Geophys. Res. Lett., 34, L02302, doi:10.1029/2006GL027586.

Vergara Munoz, A. (1988), Seismicity of the Panama Block. I: Magnitudes and spatial distribution of epicentres, Tectonophysics, 145, 213-245, doi:10.1016/0040-1951(88)90196-5.

Vogt, P. R., A. Lowrie, D. R. Bracey, and R. N. Hey (1976), Subduction of aseismic ridges: Effects on shape, seismicity, and other characteristics of consuming plate boundaries, Spec. Pap. Geol. Soc. Am., 172.

von Huene, R., C. R. Ranero, and W. Weinrebe (2000), Quaternary convergent margin tectonics of Costa Rica, segmentation of the Cocos Plate, and Central American volcanism, Tectonics, 19, 314-334, doi:10.1029/1999TC001143.

Walther, C. H. E. (2003), The crustal structure of the Cocos ridge off Costa Rica, J. Geophys. Res., 108(B3), 2136, doi:10.1029/2001JB000888.

Wang, K., R. Wells, S. Mazzotti, R. D. Hyndman, and T. Sagiya (2003), A revised dislocation model of interseismic deformation of the Cascadia subduction zone, J. Geophys. Res., 108(B1), 2026, doi:10.1029/2001JB001227.

Werner, R., K. Hoernle, P. van den Bogaard, C. Ranero, and R. von Huene (1999), Drowned 14-m.y.-old Galapagos archipelago off the coast of Costa Rica: Implications for tectonic and evolutionary models, Geology, 27, 499-502, doi:10. 1130/0091-7613(1999)027<0499:DMYOGP>2.3.CO;2.

Zumberge, J. F., M. B. Heflin, D. C. Jefferson, M. M. Watkins, and F. H. Webb (1997), Precise point positioning for the efficient and robust analysis of GPS data from large networks, J. Geophys. Res., 102, 5005-5017, doi:10.1029/96JB03860. 